SLAC-PUB-8392

USM-TH-90

hep-th/0003082

October 2000 (revised)

\title{
Light-Cone Representation of the Spin and Orbital Angular Momentum of Relativistic Composite Systems"艹
}

\author{
Stanley J. Brodsky ${ }^{a}$, Dae Sung Hwang ${ }^{b}$, Bo-Qiang $\mathrm{Ma}^{c}$, and Ivan Schmidt ${ }^{d}$ \\ ${ }^{a}$ Stanford Linear Accelerator Center, \\ Stanford University, Stanford, California 94309, USA \\ e-mail: sjbth@slac.stanford.edu \\ ${ }^{b}$ Department of Physics, Sejong University, Seoul 143-747, Korea \\ e-mail: dshwang@kunja.sejong.ac.kr \\ ${ }^{c}$ CCAST (World Laboratory), P.O. Box 8730, Beijing 100080, China, \\ Department of Physics, Peking University, Beijing 100871, China, \\ and Institute of High Energy Physics, Academia Sinica, P. O. Box 918(4), \\ Beijing 100039, China \\ e-mail: mabq@th.phy.pku.edu.cn \\ ${ }^{d}$ Departamento de Física, Universidad Técnica Federico Santa María, \\ Casilla 110-V, Valparaíso, Chile \\ e-mail: ischmidt@fis.utfsm.cl
}

\footnotetext{
*Work partially supported by the Department of Energy, contract DE-AC03-76SF00515, by the National Natural Science Foundation of China under grants No. 19605006 and No. 19975052, by Fondecyt (Chile) under grants 1990806 and 8000017, and by a Cátedra Presidencial (Chile).
} 


\begin{abstract}
The matrix elements of local operators such as the electromagnetic current, the energy momentum tensor, angular momentum, and the moments of structure functions have exact representations in terms of light-cone Fock state wavefunctions of bound states such as hadrons. We illustrate all of these properties by giving explicit light-cone wavefunctions for the two-particle Fock state of the electron in QED, thus connecting the Schwinger anomalous magnetic moment to the spin and orbital momentum carried by its Fock state constituents. We also compute the QED one-loop radiative corrections for the form factors for the graviton coupling to the electron and photon. Although the underlying model is derived from elementary QED perturbative couplings, it in fact can be used to simulate much more general bound state systems by applying spectral integration over the constituent masses while preserving all of the Lorentz properties, giving explicit realization of the spin sum rules and other local matrix elements. The role of orbital angular momentum in understanding the "spin crisis" problem for relativistic systems is clarified. We also prove that the anomalous gravitomagnetic moment $B(0)$ vanishes for any composite system. This property is shown to follow directly from the Lorentz boost properties of the lightcone Fock representation and holds separately for each Fock state component. We show how the QED perturbative structure can be used to model bound state systems while preserving all Lorentz properties. We thus obtain a theoretical laboratory to test the consistency of formulae which have been proposed to probe the spin structure of hadrons.
\end{abstract}

PACS numbers: 12.20.-m, 12.39.Ki, 13.40.Em, 13.40.Gp.

Submitted to Nucl. Phys. B 


\section{Introduction}

The light-cone Fock representation of composite systems such as hadrons in QCD has a number of remarkable properties. Because the generators of certain Lorentz boosts are kinematical, knowing the wavefunction in one frame allows one to obtain it in any other frame. Furthermore, matrix elements of space-like local operators for the coupling of photons, gravitons, and the moments of deep inelastic structure functions all can be expressed as overlaps of light-cone wavefunctions with the same number of Fock constituents. This is possible since in each case one can choose the special frame $q^{+}=0$ [1, 2] for the space-like momentum transfer and take matrix elements of "plus" components of currents such as $J^{+}$and $T^{++}$. Since the physical vacuum in light-cone quantization coincides with the perturbative vacuum, no contributions to matrix elements from vacuum fluctuations occur [3]. Light-cone Fock state wavefunctions thus encode all of the bound state quark and gluon properties of hadrons including their spin and flavor correlations in the form of universal process- and frame- independent amplitudes.

Formally, the light-cone expansion is constructed by quantizing QCD at fixed lightcone time [4] $\tau=t+z / c$ and forming the invariant light-cone Hamiltonian: $H_{L C}^{Q C D}=$

$P^{+} P^{-}-\vec{P}_{\perp}^{2}$ where $P^{ \pm}=P^{0} \pm P^{z}[3]$. The momentum generators $P^{+}$and $\vec{P}_{\perp}$ are kinematical; i.e., they are independent of the interactions. The generator $P^{-}=i \frac{d}{d \tau}$ generates light-cone time translations, and the eigen-spectrum of the Lorentz scalar $H_{L C}^{Q C D}$ gives the mass spectrum of the color-singlet hadron states in QCD together with their respective light-cone wavefunctions. For example, the proton state satisfies: $H_{L C}^{Q C D}\left|\psi_{p}\right\rangle=M_{p}^{2}\left|\psi_{p}\right\rangle$. The expansion of the proton eigensolution $\left|\psi_{p}\right\rangle$ on the colorsinglet $B=1, Q=1$ eigenstates $\{|n\rangle\}$ of the free Hamiltonian $H_{L C}^{Q C D}(g=0)$ gives the light-cone Fock expansion:

$$
\begin{aligned}
\left|\psi_{p}\left(P^{+}, \vec{P}_{\perp}\right)\right\rangle=\sum_{n} \prod_{i=1}^{n} \frac{\mathrm{d} x_{i} \mathrm{~d}^{2} \vec{k}_{\perp i}}{\sqrt{x_{i}} 16 \pi^{3}} 16 \pi^{3} \delta\left(1-\sum_{i=1}^{n} x_{i}\right) \delta^{(2)}\left(\sum_{i=1}^{n} \vec{k}_{\perp i}\right) \\
\times \psi_{n}\left(x_{i}, \vec{k}_{\perp i}, \lambda_{i}\right)\left|n ; x_{i} P^{+}, x_{i} \vec{P}_{\perp}+\vec{k}_{\perp i}, \lambda_{i}\right\rangle .
\end{aligned}
$$


The light-cone momentum fractions $x_{i}=k_{i}^{+} / P^{+}$and $\vec{k}_{\perp i}$ represent the relative momentum coordinates of the QCD constituents. The physical transverse momenta are $\vec{p}_{\perp i}=x_{i} \vec{P}_{\perp}+\vec{k}_{\perp i}$. The $\lambda_{i}$ label the light-cone spin projections $S^{z}$ of the quarks and gluons along the quantization direction $z$. The physical gluon polarization vectors $\epsilon^{\mu}(k, \lambda= \pm 1)$ are specified in light-cone gauge by the conditions $k \cdot \epsilon=0, \eta \cdot \epsilon=$ $\epsilon^{+}=0$. The $n$-particle states are normalized as

$$
\left\langle n ; p_{i}^{\prime+}, \vec{p}_{\perp i}^{\prime}, \lambda_{i}^{\prime} \mid n ; p_{i}^{+}, \vec{p}_{\perp i}, \lambda_{i}\right\rangle=\prod_{i=1}^{n} 16 \pi^{3} p_{i}^{+} \delta\left(p_{i}^{\prime+}-p_{i}^{+}\right) \delta^{(2)}\left(\vec{p}_{\perp i}^{\prime}-\vec{p}_{\perp i}\right) \delta_{\lambda_{i}^{\prime} \lambda_{i}} .
$$

The solutions of $H_{L C}^{Q C D}\left|\psi_{p}\right\rangle=M_{p}^{2}\left|\psi_{p}\right\rangle$ are independent of $P^{+}$and $\vec{P}_{\perp}$; thus given the eigensolution Fock projections $\left\langle n ; x_{i}, \vec{k}_{\perp i}, \lambda_{i} \mid \psi_{p}\right\rangle=\psi_{n}\left(x_{i}, \vec{k}_{\perp i}, \lambda_{i}\right)$, the wavefunction of the proton is determined in any frame [5]. In contrast, in equal-time quantization, a Lorentz boost always mixes dynamically with the interactions, so that computing a wavefunction in a new frame requires solving a nonperturbative problem as complicated as the Hamiltonian eigenvalue problem itself.

The LC wavefunctions $\psi_{n / H}\left(x_{i}, \vec{k}_{\perp i}, \lambda_{i}\right)$ are universal, process independent, and thus control all hadronic reactions. Given the light-cone wavefunctions, one can compute the moments of the helicity and transversity distributions measurable in polarized deep inelastic experiments [5]. For example, the polarized quark distributions at resolution $\Lambda$ correspond to

$$
\begin{aligned}
q_{\lambda_{q} / \Lambda_{p}}(x, \Lambda)= & \sum_{n, q_{a}} \int \prod_{j=1}^{n} d x_{j} d^{2} k_{\perp j} \sum_{\lambda_{i}}\left|\psi_{n / H}^{(\Lambda)}\left(x_{i}, \vec{k}_{\perp i}, \lambda_{i}\right)\right|^{2} \\
& \times \delta\left(1-\sum_{i}^{n} x_{i}\right) \delta^{(2)}\left(\sum_{i}^{n} \vec{k}_{\perp i}\right) \delta\left(x-x_{q}\right) \delta_{\lambda_{a}, \lambda_{q}} \Theta\left(\Lambda^{2}-\mathcal{M}_{n}^{2}\right),
\end{aligned}
$$

where the sum is over all quarks $q_{a}$ which match the quantum numbers, light-cone momentum fraction $x$, and helicity of the struck quark. Similarly, moments of transversity distributions and off-diagonal helicity convolutions are defined as a density matrix of the light-cone wavefunctions. Applications of non-forward quark and gluon distributions have been discussed in Refs. [6, 7]. The light-cone wavefunctions also specify the multi-quark and gluon correlations of the hadron. For example, the distribution of spectator particles in the final state which could be measured in the proton 
fragmentation region in deep inelastic scattering at an electron-proton collider are in principle encoded in the light-cone wavefunctions.

Given the $\psi_{n / H}^{(\Lambda)}$, one can construct any spacelike electromagnetic, electroweak, or gravitational form factor or local operator product matrix element of a composite or elementary system from the diagonal overlap of the LC wavefunctions [8]. Studying the gravitational form factors is not academic: Ji has shown that there is a remarkable connection of the $x$-moments of the chiral-conserving and chiral-flip form factors $H(x, t, \zeta)$ and $E(x, t, \zeta)$ which appear in deeply virtual scattering with the corresponding spin-conserving and spin-flip electromagnetic form factors $F_{1}(t)$ and $F_{2}(t)$ and gravitational form factors $A_{\mathrm{q}}(t)$ and $B_{\mathrm{q}}(t)$ for each quark and anti-quark constituent. [9] Thus, in effect, one can use virtual Compton scattering to measure graviton couplings to the charged constituents of a hadron.

Exclusive semi-leptonic $B$-decay amplitudes involving timelike currents such as $B \rightarrow A \ell \bar{\nu}$ can also be evaluated exactly in the light-cone formalism 10]. In this case, the timelike decay matrix elements require the computation of both the diagonal matrix element $n \rightarrow n$ where parton number is conserved and the off-diagonal $n+$ $1 \rightarrow n-1$ convolution such that the current operator annihilates a $q \overline{q^{\prime}}$ pair in the initial $B$ wavefunction. This term is a consequence of the fact that the time-like decay $q^{2}=\left(p_{\ell}+p_{\bar{\nu}}\right)^{2}>0$ requires a positive light-cone momentum fraction $q^{+}>0$. Conversely for space-like currents, one can choose $q^{+}=0$, as in the Drell-Yan-West representation of the space-like electromagnetic form factors [ 8 ]. However, as can be seen from the explicit analysis of timelike form factors in a perturbative model, the off-diagonal convolution can yield a non-zero $q^{+} / q^{+}$limiting form as $q^{+} \rightarrow 0$ 10. This extra term appears specifically in the case of "bad" currents such as $J^{-}$in which the coupling to $q \overline{q^{\prime}}$ fluctuations in the light-cone wavefunctions are favored [10]. In effect, the $q^{+} \rightarrow 0$ limit generates $\delta(x)$ contributions as residues of the $n+1 \rightarrow n-1$ contributions. The necessity for such "zero mode" $\delta(x)$ terms has been noted by Chang, Root and Yan [11], Burkardt [12], and Choi and Ji [13]. We can avoid these contributions by restricting our attention to the plus currents $J^{+}$and $T^{++}$. 
It should be emphasized that the light-cone Fock representation provides an exact formulation of current matrix elements of local operators. In contrast, in equal-time Hamiltonian theory, one must evaluate connected time-ordered diagrams where the gauge particle or graviton couples to particles associated with vacuum fluctuations. Thus even if one knows the equal-time wavefunction for the initial and final hadron, one cannot determine the current matrix elements. In the case of the covariant BetheSalpeter formalism, the evaluation of the matrix element of the current requires the calculation of an infinite number of irreducible diagram contributions.

One of the important issues in the formulation of light-cone quantized quantum field theories is the existence of a consistent scheme for non-perturbative renormalization. A general nonperturbative renormalization procedure for QCD has recently been outlined by Paston et al. [14]. An alternative is to the use of broken supersymmetry as an ultraviolet regulator [15]. Some simplified model light-cone field theories have been successfully renormalized using generalized Pauli-Villars regularization [15].

As an illustration of the structure of the light-cone Fock state representation, we will present a simple self-consistent model of an effective composite spin- $\frac{1}{2}$ system based on the quantum fluctuations of the electron in QED. The model is patterned after the structure which occurs in the one-loop Schwinger $\alpha / 2 \pi$ correction to the electron magnetic moment [8]. In effect, we can represent a spin- $\frac{1}{2}$ system as a composite of a spin- $\frac{1}{2}$ fermion and spin-one vector boson with arbitrary masses. We also give results for the case of a spin- $\frac{1}{2}$ composite consisting of scalar plus spin- $\frac{1}{2}$ constituents, as would occur in a composite of a photino and slepton in supersymmetric QED and in the radiative corrections due to Higgs exchange. The light-cone wavefunctions describe off-shell particles but are computable explicitly from perturbation theory. We will explicitly compute the form factors $F_{1}\left(q^{2}\right)$ and $F_{2}\left(q^{2}\right)$ of the electromagnetic current, and the various contributions to the form factors $A\left(q^{2}\right)$ and $B\left(q^{2}\right)$ of the energy-momentum tensor. The model thus provides a check on the general formulae, particularly the structure of angular momentum on the light-cone; it provides an important illustration of $J^{z}$ conservation, Fock state by Fock state; it 
demonstrates helicity retention between fermions and vector bosons at $x \rightarrow 1$; and it provides a template for an effective quark spin-one diquark structure of the valence light-cone wavefunction of the proton.

The one-loop models can be further generalized by applying spectral Pauli-Villars integration over the constituent masses. This representation of an effectively composite system is particularly useful because it is based simply on two constituents but yet is totally relativistic. The resulting form of light-cone wavefunctions provides a template for parameterizing the structure of relativistic composite systems and their matrix elements in hadronic physics. We thus obtain a theoretical laboratory to test the consistency of formulae which have been proposed to probe the spin structure of hadrons. This clarifies the connection of parton distributions to the constituents' spin and orbital angular momentum and to static quantities of the composite systems such as the magnetic moment. For example, the model also provides a self-consistent form for the wavefunctions of an effective quark-diquark model of the valence Fock state of the proton wavefunction. A similar approach has recently been used to illustrate the evolution of light-cone helicity and orbital angular momentum operators [16]. A nonperturbative calculation of the electron magnetic moment using the discretized light-cone quantization method [3] is given in Ref. [17].

Many of the features of the analysis apply to arbitrary composite systems. For example, we will explicitly prove the vanishing of the anomalous gravito-moment coupling $B(0)$ to gravity for any composite system. This remarkable result was first derived classically from the equivalence principle by Okun and Kobsarev [18, and from the conservation of the energy-momentum tensor by Kobsarev and Zakharov [19. See also the more recent discussions in Refs. [9, 20]. We will demonstrate that $B(0)=0$ follows directly for composite systems in quantum field theory from the Lorentz boost properties of the light-cone Fock representation, and that it is valid in every Fock sector. 


\section{Electromagnetic and Gravitational Form Factors}

The light-cone Fock representation allows one to compute all matrix elements of local currents as overlap integrals of the light-cone Fock wavefunctions. In particular, we can evaluate the forward and non-forward matrix elements of the electroweak currents, moments of the deep inelastic structure functions, as well as the electromagnetic form factors and the magnetic moment. Given the local operators for the energy-momentum tensor $T^{\mu \nu}(x)$ and the angular momentum tensor $M^{\mu \nu \lambda}(x)$, one can directly compute momentum fractions, spin properties, the gravitomagnetic moment, and the form factors $A\left(q^{2}\right)$ and $B\left(q^{2}\right)$ appearing in the coupling of gravitons to composite systems.

In the case of a spin- $\frac{1}{2}$ composite system, the Dirac and Pauli form factors $F_{1}\left(q^{2}\right)$ and $F_{2}\left(q^{2}\right)$ are defined by

$$
\left\langle P^{\prime}\left|J^{\mu}(0)\right| P\right\rangle=\bar{u}\left(P^{\prime}\right)\left[F_{1}\left(q^{2}\right) \gamma^{\mu}+F_{2}\left(q^{2}\right) \frac{i}{2 M} \sigma^{\mu \alpha} q_{\alpha}\right] u(P),
$$

where $q^{\mu}=\left(P^{\prime}-P\right)^{\mu}$ and $u(P)$ is the bound state spinor. In the light-cone formalism it is convenient to identify the Dirac and Pauli form factors from the helicityconserving and helicity-flip vector current matrix elements of the $J^{+}$current [8]:

$$
\begin{gathered}
\left\langle P+q, \uparrow\left|\frac{J^{+}(0)}{2 P^{+}}\right| P, \uparrow\right\rangle=F_{1}\left(q^{2}\right), \\
\left\langle P+q, \uparrow\left|\frac{J^{+}(0)}{2 P^{+}}\right| P, \downarrow\right\rangle=-\left(q^{1}-\mathrm{i} q^{2}\right) \frac{F_{2}\left(q^{2}\right)}{2 M} .
\end{gathered}
$$

The magnetic moment of a composite system is one of its most basic properties. The magnetic moment is defined at the $q^{2} \rightarrow 0$ limit,

$$
\mu=\frac{e}{2 M}\left[F_{1}(0)+F_{2}(0)\right]
$$

where $e$ is the charge and $M$ is the mass of the composite system. We use the standard light-cone frame $\left(q^{ \pm}=q^{0} \pm q^{3}\right)$ :

$$
\begin{aligned}
q & =\left(q^{+}, q^{-}, \vec{q}_{\perp}\right)=\left(0, \frac{-q^{2}}{P^{+}}, \vec{q}_{\perp}\right), \\
P & =\left(P^{+}, P^{-}, \vec{P}_{\perp}\right)=\left(P^{+}, \frac{M^{2}}{P^{+}}, \overrightarrow{0}_{\perp}\right),
\end{aligned}
$$


where $q^{2}=-2 P \cdot q=-\vec{q}_{\perp}^{2}$ is 4 -momentum square transferred by the photon.

The Pauli form factor and the anomalous magnetic moment $\kappa=\frac{e}{2 M} F_{2}(0)$ can then be calculated from the expression

$$
-\left(q^{1}-\mathrm{i} q^{2}\right) \frac{F_{2}\left(q^{2}\right)}{2 M}=\sum_{a} \int \frac{\mathrm{d}^{2} \vec{k}_{\perp} \mathrm{d} x}{16 \pi^{3}} \sum_{j} e_{j} \psi_{a}^{\uparrow *}\left(x_{i}, \vec{k}_{\perp i}^{\prime}, \lambda_{i}\right) \psi_{a}^{\downarrow}\left(x_{i}, \vec{k}_{\perp i}, \lambda_{i}\right),
$$

where the summation is over all contributing Fock states $a$ and struck constituent charges $e_{j}$. The arguments of the final-state light-cone wavefunction are [1], 2]

$$
\vec{k}_{\perp i}^{\prime}=\vec{k}_{\perp i}+\left(1-x_{i}\right) \vec{q}_{\perp}
$$

for the struck constituent and

$$
\vec{k}_{\perp i}^{\prime}=\vec{k}_{\perp i}-x_{i} \vec{q}_{\perp}
$$

for each spectator. Notice that the magnetic moment must be calculated from the spin-flip non-forward matrix element of the current. It is not given by a diagonal forward matrix element 21]. In the ultra-relativistic limit where the radius of the system is small compared to its Compton scale $1 / M$, the anomalous magnetic moment must vanish [22]. The light-cone formalism is consistent with this theorem.

The form factors of the energy-momentum tensor for a spin- $\frac{1}{2}$ composite are defined by

$$
\begin{array}{r}
\left\langle P^{\prime}\left|T^{\mu \nu}(0)\right| P\right\rangle=\bar{u}\left(P^{\prime}\right)\left[A\left(q^{2}\right) \gamma^{(\mu} \bar{P}^{\nu)}+B\left(q^{2}\right) \frac{i}{2 M} \bar{P}^{(\mu} \sigma^{\nu) \alpha} q_{\alpha}\right. \\
\left.+C\left(q^{2}\right) \frac{1}{M}\left(q^{\mu} q^{\nu}-g^{\mu \nu} q^{2}\right)\right] u(P),
\end{array}
$$

where $q^{\mu}=\left(P^{\prime}-P\right)^{\mu}, \bar{P}^{\mu}=\frac{1}{2}\left(P^{\prime}+P\right)^{\mu}, a^{(\mu} b^{\nu)}=\frac{1}{2}\left(a^{\mu} b^{\nu}+a^{\nu} b^{\mu}\right)$, and $u(P)$ is the spinor of the system.

As in the light-cone decomposition Eqs. (5) and (6) of the Dirac and Pauli form factors for the vector current [8], we can obtain the light-cone representation of the $A\left(q^{2}\right)$ and $B\left(q^{2}\right)$ form factors of the energy-tensor Eq. (12). Since we work in the interaction picture, only the non-interacting parts of the energy momentum tensor 
$T^{++}(0)$ need to be computed in the light-cone formalism. By calculating the ++ component of Eq. (12), we find

$$
\begin{gathered}
\left\langle P+q, \uparrow\left|\frac{T^{++}(0)}{2\left(P^{+}\right)^{2}}\right| P, \uparrow\right\rangle=A\left(q^{2}\right), \\
\left\langle P+q, \uparrow\left|\frac{T^{++}(0)}{2\left(P^{+}\right)^{2}}\right| P, \downarrow\right\rangle=-\left(q^{1}-\mathrm{i} q^{2}\right) \frac{B\left(q^{2}\right)}{2 M} .
\end{gathered}
$$

The $A\left(q^{2}\right)$ and $B\left(q^{2}\right)$ form factors Eqs. (13) and (14) are similar to the $F_{1}\left(q^{2}\right)$ and $F_{2}\left(q^{2}\right)$ form factors Eqs. (5) and (6) with an additional factor of the light-cone momentum fraction $x=k^{+} / P^{+}$of the struck constituent in the integrand. The $B\left(q^{2}\right)$ form factor is obtained from the non-forward spin-flip amplitude. The value of $B(0)$ is obtained in the $q^{2} \rightarrow 0$ limit. The angular momentum projection of a state is given by

$$
\left\langle J^{i}\right\rangle=\frac{1}{2} \epsilon^{i j k} \int d^{3} x\left\langle T^{0 k} x^{j}-T^{0 j} x^{k}\right\rangle=A(0)\left\langle L^{i}\right\rangle+[A(0)+B(0)] \bar{u}(P) \frac{1}{2} \sigma^{i} u(P) .
$$

This result is derived using a wave packet description of the state. The $\left\langle L^{i}\right\rangle$ term is the orbital angular momentum of the center of mass motion with respect to an arbitrary origin and can be dropped. The coefficient of the $\left\langle L^{i}\right\rangle$ term must be 1; $A(0)=1$ also follows when we evaluate the four-momentum expectation value $\left\langle P^{\mu}\right\rangle$. Thus the total intrinsic angular momentum $J^{z}$ of a nucleon can be identified with the values of the form factors $A\left(q^{2}\right)$ and $B\left(q^{2}\right)$ at $q^{2}=0$ :

$$
\left\langle J^{z}\right\rangle=\left\langle\frac{1}{2} \sigma^{z}\right\rangle[A(0)+B(0)]
$$

One can define individual quark and gluon contributions to the total angular momentum from the matrix elements of the energy momentum tensor [9]. However, this definition is only formal; $A_{q, g}(0)$ can be interpreted as the light-cone momentum fraction carried by the quarks or gluons $\left\langle x_{q, g}\right\rangle$. The contributions from $B_{q, g}(0)$ to $J_{z}$ cancel in the sum. In fact, we shall show that the contributions to $B(0)$ vanish when summed over the constituents of each individual Fock state. 
We will give an explicit realization of these relations in the light-cone Fock representation for general composite systems. In the next section we will illustrate the formulae by computing the electron's electromagnetic and energy-momentum tensor form factors to one-loop order in QED. In fact, the structure of this calculation has much more generality and can be used as a template for more general composite systems.

\section{The Light-Cone Fock State Decomposition and Spin Structure of Leptons in QED}

The Schwinger one-loop radiative correction to the electron current in quantum electrodynamics has played a historic role in the development of quantum field theory. In the language of light-cone quantization, the electron anomalous magnetic moment $a_{e}=\alpha / 2 \pi$ is due to the one-fermion one-gauge boson Fock state component of the physical electron. An explicit calculation of the anomalous moment in this framework using equation (7) was give in Ref. [8]. We shall show here that the light-cone wavefunctions of the electron provides an ideal system to check explicitly the intricacies of spin and angular momentum in quantum field theory. In particular, we shall evaluate the matrix elements of the QED energy momentum tensor and show how the "spin crisis" is resolved in QED for an actual physical system. The analysis is exact in perturbation theory. The same method can be applied to the moments of structure functions and the evaluation of other local matrix elements. In fact, the QED analysis of this section is more general than perturbation theory. We will also show how the perturbative light-cone wavefunctions of leptons and photons provide a template for the wavefunctions of non-perturbative composite systems resembling hadrons in QCD.

The light-cone Fock state wavefunctions of an electron can be systematically eval- 
uated in QED. The QED Lagrangian density is

$$
\mathcal{L}=\frac{i}{2}\left[\bar{\psi} \gamma^{\mu}\left(\vec{\partial}_{\mu}+i e A_{\mu}\right) \psi-\bar{\psi} \gamma^{\mu}\left(\overleftarrow{\partial}_{\mu}-i e A_{\mu}\right) \psi\right]-m \bar{\psi} \psi-\frac{1}{4} F^{\mu \nu} F_{\mu \nu}
$$

and the corresponding energy-momentum tensor is

$$
\begin{aligned}
T^{\mu \nu}= & \frac{i}{4}\left(\left[\bar{\psi} \gamma^{\mu}\left(\vec{\partial}^{\nu}+i e A^{\nu}\right) \psi-\bar{\psi} \gamma^{\mu}\left(\overleftarrow{\partial}^{\nu}-i e A^{\nu}\right) \psi\right]+[\mu \longleftrightarrow \nu]\right) \\
& +F^{\mu \rho} F_{\rho}{ }^{\nu}+\frac{1}{4} g^{\mu \nu} F^{\rho \lambda} F_{\rho \lambda} .
\end{aligned}
$$

Since $T^{\mu \nu}$ is the Noether current of the general coordinate transformation, it is conserved. In later calculations we will identify the two terms in Eq. (18) as the fermion and boson contributions $T_{\mathrm{f}}^{\mu \nu}$ and $T_{\mathrm{b}}^{\mu \nu}$, respectively.

The physical electron is the eigenstate of the QED Hamiltonian. As discussed in the introduction, the expansion of it is the QED eigenfunction on the complete set $|n\rangle$ of $H_{0}$ eigenstates produces the Fock state expansion. It is particularly advantageous to carry out this procedure using light-cone quantization since the vacuum is trivial, the Fock state representation is boost invariant, and the light-cone fractions $x_{i}=k_{i}^{+} / P^{+}$ are positive: $0<x_{i} \leq 1, \sum_{i} x_{i}=1$. We also employ light-cone gauge $A^{+}=0$ so that the gauge boson polarizations are physical. Thus each Fock-state wavefunction $\langle n|$ physical electron $\rangle$ of the physical electron with total spin projection $J^{z}= \pm \frac{1}{2}$ is represented by the function $\psi_{n}^{J^{z}}\left(x_{i}, \vec{k}_{\perp i}, \lambda_{i}\right)$, where

$$
k_{i}=\left(k_{i}^{+}, k_{i}^{-}, \vec{k}_{\perp i}\right)=\left(x_{i} P^{+}, \frac{\vec{k}_{\perp i}^{2}+m_{i}^{2}}{x_{i} P^{+}}, \vec{k}_{\perp i}\right)
$$

specifies the momentum of each constituent and $\lambda_{i}$ specifies its light-cone helicity in the $z$ direction. We adopt a non-zero boson mass $\lambda$ for the sake of generality.

The two-particle Fock state for an electron with $J^{z}=+\frac{1}{2}$ has four possible spin combinations:

$$
\begin{aligned}
& \left|\Psi_{\text {two particle }}^{\uparrow}\left(P^{+}, \vec{P}_{\perp}=\overrightarrow{0}_{\perp}\right)\right\rangle=\int \frac{\mathrm{d}^{2} \vec{k}_{\perp} \mathrm{d} x}{\sqrt{x(1-x)} 16 \pi^{3}} \\
& \times \quad\left[\psi_{+\frac{1}{2}+1}^{\uparrow}\left(x, \vec{k}_{\perp}\right)\left|+\frac{1}{2}+1 ; x P^{+}, \vec{k}_{\perp}\right\rangle+\psi_{+\frac{1}{2}-1}^{\uparrow}\left(x, \vec{k}_{\perp}\right)\left|+\frac{1}{2}-1 ; x P^{+}, \vec{k}_{\perp}\right\rangle\right. \\
& \left.\quad+\psi_{-\frac{1}{2}+1}^{\uparrow}\left(x, \vec{k}_{\perp}\right)\left|-\frac{1}{2}+1 ; x P^{+}, \vec{k}_{\perp}\right\rangle+\psi_{-\frac{1}{2}-1}^{\uparrow}\left(x, \vec{k}_{\perp}\right)\left|-\frac{1}{2}-1 ; x P^{+}, \vec{k}_{\perp}\right\rangle\right],
\end{aligned}
$$


where the two-particle states $\left|s_{\mathrm{f}}^{z}, s_{\mathrm{b}}^{z} ; x P^{+}, \vec{k}_{\perp}\right\rangle$ are normalized as in (2). Here $s_{\mathrm{f}}^{z}$ and $s_{\mathrm{b}}^{z}$ denote the $z$-component of the spins of the constituent fermion and boson, respectively. The wavefunctions can be evaluated explicitly in QED perturbation theory using the rules given in Refs. [5, 8]:

$$
\left\{\begin{array}{l}
\psi_{+\frac{1}{2}+1}^{\uparrow}\left(x, \vec{k}_{\perp}\right)=-\sqrt{2} \frac{\left(-k^{1}+\mathrm{i} k^{2}\right)}{x(1-x)} \varphi \\
\psi_{+\frac{1}{2}-1}^{\uparrow}\left(x, \vec{k}_{\perp}\right)=-\sqrt{2} \frac{\left(+k^{1}+\mathrm{i} k^{2}\right)}{1-x} \varphi \\
\psi_{-\frac{1}{2}+1}^{\uparrow}\left(x, \vec{k}_{\perp}\right)=-\sqrt{2}\left(M-\frac{m}{x}\right) \varphi \\
\psi_{-\frac{1}{2}-1}^{\uparrow}\left(x, \vec{k}_{\perp}\right)=0
\end{array}\right.
$$

where

$$
\varphi=\varphi\left(x, \vec{k}_{\perp}\right)=\frac{e / \sqrt{1-x}}{M^{2}-\left(\vec{k}_{\perp}^{2}+m^{2}\right) / x-\left(\vec{k}_{\perp}^{2}+\lambda^{2}\right) /(1-x)} .
$$

Similarly,

$$
\begin{gathered}
\left|\Psi_{\text {two particle }}^{\downarrow}\left(P^{+}, \vec{P}_{\perp}=\overrightarrow{0}_{\perp}\right)\right\rangle=\int \frac{\mathrm{d}^{2} \vec{k}_{\perp} \mathrm{d} x}{\sqrt{x(1-x)} 16 \pi^{3}} \\
\times \quad\left[\psi_{+\frac{1}{2}+1}^{\downarrow}\left(x, \vec{k}_{\perp}\right)\left|+\frac{1}{2}+1 ; x P^{+}, \vec{k}_{\perp}\right\rangle+\psi_{+\frac{1}{2}-1}^{\downarrow}\left(x, \vec{k}_{\perp}\right)\left|+\frac{1}{2}-1 ; x P^{+}, \vec{k}_{\perp}\right\rangle\right. \\
\left.\quad+\psi_{-\frac{1}{2}+1}^{\downarrow}\left(x, \vec{k}_{\perp}\right)\left|-\frac{1}{2}+1 ; x P^{+}, \vec{k}_{\perp}\right\rangle+\psi_{-\frac{1}{2}-1}^{\downarrow}\left(x, \vec{k}_{\perp}\right)\left|-\frac{1}{2}-1 ; x P^{+}, \vec{k}_{\perp}\right\rangle\right],
\end{gathered}
$$

where

$$
\left\{\begin{array}{l}
\psi_{+\frac{1}{2}+1}^{\downarrow}\left(x, \vec{k}_{\perp}\right)=0, \\
\psi_{+\frac{1}{2}-1}^{\downarrow}\left(x, \vec{k}_{\perp}\right)=-\sqrt{2}\left(M-\frac{m}{x}\right) \varphi, \\
\psi_{-\frac{1}{2}+1}^{\downarrow}\left(x, \vec{k}_{\perp}\right)=-\sqrt{2} \frac{\left(-k^{1}+\mathrm{i} k^{2}\right)}{1-x} \varphi \\
\psi_{-\frac{1}{2}-1}^{\downarrow}\left(x, \vec{k}_{\perp}\right)=-\sqrt{2} \frac{\left(+k^{1}+\mathrm{i} k^{2}\right)}{x(1-x)} \varphi .
\end{array}\right.
$$

The coefficients of $\varphi$ in Eqs. (21) and (24) are the matrix elements of $\frac{\bar{u}\left(k^{+}, k^{-}, \vec{k}_{\perp}\right)}{\sqrt{k^{+}}} \gamma$. $\epsilon^{*} \frac{u\left(P^{+}, P^{-}, \vec{P}_{\perp}\right)}{\sqrt{P^{+}}}$which are the numerators of the wavefunctions corresponding to each constituent spin $s^{z}$ configuration. The two boson polarization vectors in light-cone gauge are $\epsilon^{\mu}=\left(\epsilon^{+}=0, \epsilon^{-}=\frac{\vec{\epsilon}_{\perp} \cdot \vec{k}_{\perp}}{2 k^{+}}, \vec{\epsilon}_{\perp}\right)$ where $\vec{\epsilon}=\overrightarrow{\epsilon_{\perp \uparrow, \downarrow}}=\mp(1 / \sqrt{2})(\widehat{x} \pm \mathrm{i} \widehat{y})$. The polarizations also satisfy the Lorentz condition $k \cdot \epsilon=0$. In (21) and (24) we have generalized the framework of QED by assigning a mass $M$ to the external electrons, 
but a different mass $m$ to the internal electron lines and a mass $\lambda$ to the internal photon line [8]. The idea behind this is to model the structure of a composite fermion state with mass $M$ by a fermion and a vector constituent with respective masses $m$ and $\lambda$.

The electron in QED also has a "bare" one-particle component:

$$
\left|\Psi_{\text {one particle }}^{\uparrow, \downarrow}\right\rangle=\sqrt{Z} \delta(1-x) \delta\left(\vec{k}_{\perp}=\overrightarrow{0}_{\perp}\right)\left|s_{\mathrm{f}}^{z}= \pm \frac{1}{2}\right\rangle
$$

where $Z$ is the wavefunction normalization of the one-particle state. If we regulate the theory in the ultraviolet and infrared, $Z$ is finite.

We first will evaluate the Dirac and Pauli form factors $F_{1}\left(q^{2}\right)$ and $F_{2}\left(q^{2}\right)$. Using Eqs. (5) and (20) we have to order $e^{2}$

$$
\begin{gathered}
F_{1}\left(q^{2}\right)=\left\langle\Psi^{\uparrow}\left(P^{+}, \vec{P}_{\perp}=\vec{q}_{\perp}\right)\right)\left|\Psi^{\uparrow}\left(P^{+}, \vec{P}_{\perp}=\overrightarrow{0}_{\perp}\right)\right\rangle \\
=Z+\int \frac{\mathrm{d}^{2} \vec{k}_{\perp} \mathrm{d} x}{16 \pi^{3}}\left[\psi_{+\frac{1}{2}+1}^{\uparrow *}\left(x, \vec{k}_{\perp}^{\prime}\right) \psi_{+\frac{1}{2}+1}^{\uparrow}\left(x, \vec{k}_{\perp}\right)+\psi_{+\frac{1}{2}-1}^{\uparrow *}\left(x, \vec{k}_{\perp}^{\prime}\right) \psi_{+\frac{1}{2}-1}^{\uparrow}\left(x, \vec{k}_{\perp}\right)\right. \\
\left.+\psi_{-\frac{1}{2}+1}^{\uparrow *}\left(x, \vec{k}_{\perp}^{\prime}\right) \psi_{-\frac{1}{2}+1}^{\uparrow}\left(x, \vec{k}_{\perp}\right)\right],
\end{gathered}
$$

where

$$
\vec{k}_{\perp}^{\prime}=\vec{k}_{\perp}+(1-x) \vec{q}_{\perp}
$$

Ultraviolet regularization is assumed. For example, we can assume a cutoff in the invariant mass of the constituents: $\mathcal{M}^{2}=\sum_{i} \frac{\vec{k}_{\perp i}^{2}+m_{i}^{2}}{x_{i}}<\Lambda^{2}$, or we can use PauliVillars regularization by introducing a fictitious photon with a large mass $\Lambda$.

At zero momentum transfer

$$
\begin{gathered}
F_{1}(0)=Z+\int \frac{\mathrm{d}^{2} \vec{k}_{\perp} \mathrm{d} x}{16 \pi^{3}}\left[\psi_{+\frac{1}{2}+1}^{\uparrow *}\left(x, \vec{k}_{\perp}\right) \psi_{+\frac{1}{2}+1}^{\uparrow}\left(x, \vec{k}_{\perp}\right)+\psi_{+\frac{1}{2}-1}^{\uparrow *}\left(x, \vec{k}_{\perp}\right) \psi_{+\frac{1}{2}-1}^{\uparrow}\left(x, \vec{k}_{\perp}\right)\right. \\
\left.+\psi_{-\frac{1}{2}+1}^{\uparrow *}\left(x, \vec{k}_{\perp}\right) \psi_{-\frac{1}{2}+1}^{\uparrow}\left(x, \vec{k}_{\perp}\right)\right]
\end{gathered}
$$

where the renormalization constant $Z$ is given, using Pauli-Villars regularization, by

$$
\begin{aligned}
Z=1- & \frac{\alpha}{2 \pi} \int_{0}^{1} d x\left[\frac{\left(1+x^{2}\right)}{(1-x)} \ln \frac{-M^{2}+\frac{m^{2}}{x}+\frac{\Lambda^{2}}{1-x}}{-M^{2}+\frac{m^{2}}{x}+\frac{\lambda^{2}}{1-x}}\right. \\
& \left.+\frac{(-x M+m)^{2}}{x}\left(-\frac{1}{-M^{2}+\frac{m^{2}}{x}+\frac{\Lambda^{2}}{1-x}}+\frac{1}{-M^{2}+\frac{m^{2}}{x}+\frac{\lambda^{2}}{1-x}}\right)\right] .
\end{aligned}
$$


This ensures the Ward identity and $F_{1}(0)=1$. Further discussion of the Ward identity for QED in light-cone perturbation theory is given in ref. 23]

The one-loop model can be further generalized by applying spectral Pauli-Villars integration over the constituent masses. The resulting form of light-cone wavefunctions provides a template for parameterizing the structure of relativistic composite systems and their matrix elements in hadronic physics.

The Pauli form factor is obtained from the spin-flip matrix element of the $J^{+}$ current. From Eqs. (6), (20), and (23) we have

$$
\begin{aligned}
F_{2}\left(q^{2}\right)= & \frac{-2 M}{\left(q^{1}-\mathrm{i} q^{2}\right)}\left\langle\Psi^{\uparrow}\left(P^{+}, \vec{P}_{\perp}=\vec{q}_{\perp}\right)\right)\left|\Psi^{\downarrow}\left(P^{+}, \vec{P}_{\perp}=\overrightarrow{0}_{\perp}\right)\right\rangle \\
= & \frac{-2 M}{\left(q^{1}-\mathrm{i} q^{2}\right)} \int \frac{\mathrm{d}^{2} \vec{k}_{\perp} \mathrm{d} x}{16 \pi^{3}}\left[\psi_{+\frac{1}{2}-1}^{\uparrow *}\left(x, \vec{k}_{\perp}^{\prime}\right) \psi_{+\frac{1}{2}-1}^{\downarrow}\left(x, \vec{k}_{\perp}\right)+\psi_{-\frac{1}{2}+1}^{\uparrow *}\left(x, \vec{k}_{\perp}^{\prime}\right) \psi_{-\frac{1}{2}+1}^{\downarrow}\left(x, \vec{k}_{\perp}\right)\right] \\
= & 4 M \int \frac{\mathrm{d}^{2} \vec{k}_{\perp} \mathrm{d} x}{16 \pi^{3}} \frac{(m-M x)}{x} \varphi\left(x, \vec{k}_{\perp}^{\prime}\right)^{*} \varphi\left(x, \vec{k}_{\perp}\right) \\
= & 4 M e^{2} \int \frac{\mathrm{d}^{2} \vec{k}_{\perp} \mathrm{d} x}{16 \pi^{3}} \frac{(m-x M)}{x(1-x)} \\
& \times \frac{1}{\left[M^{2}-\left(\left(\vec{k}_{\perp}+(1-x) \vec{q}_{\perp}\right)^{2}+m^{2}\right) / x-\left(\left(\vec{k}_{\perp}+(1-x) \vec{q}_{\perp}\right)^{2}+\lambda^{2}\right) /(1-x)\right]} \\
& \times \frac{1}{\left[M^{2}-\left(\vec{k}_{\perp}^{2}+m^{2}\right) / x-\left(\vec{k}_{\perp}^{2}+\lambda^{2}\right) /(1-x)\right]} .
\end{aligned}
$$

Using the Feynman parameterization, we can also express Eq. (30) in a form in which the $q^{2}=-\vec{q}_{\perp}^{2}$ dependence is more explicit as

$$
F_{2}\left(q^{2}\right)=\frac{M e^{2}}{4 \pi^{2}} \int_{0}^{1} d \alpha \int_{0}^{1} d x \frac{m-x M}{\alpha(1-\alpha) \frac{1-x}{x} \vec{q}_{\perp}^{2}-M^{2}+\frac{m^{2}}{x}+\frac{\lambda^{2}}{1-x}} .
$$

The anomalous moment is obtained in the limit of zero momentum transfer:

$$
\begin{aligned}
F_{2}(0) & =4 M e^{2} \int \frac{\mathrm{d}^{2} \vec{k}_{\perp} \mathrm{d} x}{16 \pi^{3}} \frac{(m-x M)}{x(1-x)} \frac{1}{\left[M^{2}-\left(\vec{k}_{\perp}^{2}+m^{2}\right) / x-\left(\vec{k}_{\perp}^{2}+\lambda^{2}\right) /(1-x)\right]^{2}} \\
& =\frac{M e^{2}}{4 \pi^{2}} \int_{0}^{1} d x \frac{m-x M}{-M^{2}+\frac{m^{2}}{x}+\frac{\lambda^{2}}{1-x}}
\end{aligned}
$$

which is the result of Ref. [8]. For zero photon mass and $M=m$, it gives the correct order $\alpha$ Schwinger value $a_{e}=F_{2}(0)=\alpha / 2 \pi$ for the electron anomalous magnetic moment for QED. 
As seen from Eqs. (13) and (14), the matrix elements of the double plus components of the energy-momentum tensor are sufficient to derive the fermion and boson constituents' form factors $A_{\mathrm{f}, \mathrm{g}}\left(q^{2}\right)$ and $B_{\mathrm{f}, \mathrm{g}}\left(q^{2}\right)$ of graviton coupling to matter. In particular, we shall verify $A(0)=A_{\mathrm{f}}(0)+A_{\mathrm{b}}(0)=1$ and $B(0)=0$.

The individual contributions of the fermion and boson fields to the energy-momentum form factors in QED are given by

$$
\begin{gathered}
A_{\mathrm{f}}\left(q^{2}\right)=\left\langle\Psi^{\uparrow}\left(P^{+}, \vec{P}_{\perp}=\vec{q}_{\perp}\right)\left|\frac{T_{\mathrm{f}}^{++}(0)}{2\left(P^{+}\right)^{2}}\right| \Psi^{\uparrow}\left(P^{+}, \vec{P}_{\perp}=\overrightarrow{0}_{\perp}\right)\right\rangle \\
=\int \frac{\mathrm{d}^{2} \vec{k}_{\perp} \mathrm{d} x}{16 \pi^{3}} x\left[\begin{array}{c}
\psi_{+\frac{1}{2}+1}^{\uparrow *}\left(x, \vec{k}_{\perp}^{\prime}\right) \psi_{+\frac{1}{2}+1}^{\uparrow}\left(x, \vec{k}_{\perp}\right)+\psi_{+\frac{1}{2}-1}^{\uparrow *}\left(x, \vec{k}_{\perp}^{\prime}\right) \psi_{+\frac{1}{2}-1}^{\uparrow}\left(x, \vec{k}_{\perp}\right) \\
\left.+\psi_{-\frac{1}{2}+1}^{\uparrow *}\left(x, \vec{k}_{\perp}^{\prime}\right) \psi_{-\frac{1}{2}+1}^{\uparrow}\left(x, \vec{k}_{\perp}\right)\right]
\end{array}\right.
\end{gathered}
$$

where $\vec{k}_{\perp}^{\prime}$ is given in Eq. (27), and

$$
\begin{gathered}
A_{\mathrm{b}}\left(q^{2}\right)=\left\langle\Psi^{\uparrow}\left(P^{+}, \vec{P}_{\perp}=\vec{q}_{\perp}\right)\left|\frac{T_{\mathrm{b}}^{++}(0)}{2\left(P^{+}\right)^{2}}\right| \Psi^{\uparrow}\left(P^{+}, \vec{P}_{\perp}=\overrightarrow{0}_{\perp}\right)\right\rangle \\
=\int \frac{\mathrm{d}^{2} \vec{k}_{\perp} \mathrm{d} x}{16 \pi^{3}}(1-x)\left[\psi_{+\frac{1}{2}+1}^{\uparrow *}\left(x, \vec{k}_{\perp}^{\prime \prime}\right) \psi_{+\frac{1}{2}+1}^{\uparrow}\left(x, \vec{k}_{\perp}\right)+\psi_{+\frac{1}{2}-1}^{\uparrow *}\left(x, \vec{k}_{\perp}^{\prime \prime}\right) \psi_{+\frac{1}{2}-1}^{\uparrow}\left(x, \vec{k}_{\perp}\right)\right. \\
\left.+\psi_{-\frac{1}{2}+1}^{\uparrow *}\left(x, \vec{k}_{\perp}^{\prime \prime}\right) \psi_{-\frac{1}{2}+1}^{\uparrow}\left(x, \vec{k}_{\perp}\right)\right],
\end{gathered}
$$

where

$$
\vec{k}_{\perp}^{\prime \prime}=\vec{k}_{\perp}-x \vec{q}_{\perp} .
$$

Note that

$$
A_{\mathrm{f}}(0)+A_{\mathrm{b}}(0)=F_{1}(0)=1,
$$

which corresponds to the momentum sum rule.

The fermion and boson contributions to the spin-flip matter form factor are

$$
\begin{aligned}
& B_{\mathrm{f}}\left(q^{2}\right)= \frac{-2 M}{\left(q^{1}-\mathrm{i} q^{2}\right)}\left\langle\Psi^{\uparrow}\left(P^{+}, \vec{P}_{\perp}=\vec{q}_{\perp}\right)\left|\frac{T_{\mathrm{f}}^{++}(0)}{2\left(P^{+}\right)^{2}}\right| \Psi^{\downarrow}\left(P^{+}, \vec{P}_{\perp}=\overrightarrow{0}_{\perp}\right)\right\rangle \\
&= \frac{-2 M}{\left(q^{1}-\mathrm{i} q^{2}\right)} \int \frac{\mathrm{d}^{2} \vec{k}_{\perp} \mathrm{d} x}{16 \pi^{3}} x \\
& \quad \times\left[\psi_{+\frac{1}{2}-1}^{\uparrow *}\left(x, \vec{k}_{\perp}^{\prime}\right) \psi_{+\frac{1}{2}-1}^{\downarrow}\left(x, \vec{k}_{\perp}\right)+\psi_{-\frac{1}{2}+1}^{\uparrow *}\left(x, \vec{k}_{\perp}^{\prime}\right) \psi_{-\frac{1}{2}+1}^{\downarrow}\left(x, \vec{k}_{\perp}\right)\right] \\
&= 4 M \int \frac{\mathrm{d}^{2} \vec{k}_{\perp} \mathrm{d} x}{16 \pi^{3}}(m-M x) \varphi\left(x, \vec{k}_{\perp}^{\prime}\right)^{*} \varphi\left(x, \vec{k}_{\perp}\right)
\end{aligned}
$$




$$
\begin{aligned}
= & 4 M e^{2} \int \frac{\mathrm{d}^{2} \vec{k}_{\perp} \mathrm{d} x}{16 \pi^{3}} \frac{(m-x M)}{(1-x)} \\
& \times \frac{1}{\left[M^{2}-\left(\left(\vec{k}_{\perp}+(1-x) \vec{q}_{\perp}\right)^{2}+m^{2}\right) / x-\left(\left(\vec{k}_{\perp}+(1-x) \vec{q}_{\perp}\right)^{2}+\lambda^{2}\right) /(1-x)\right]} \\
& \times \frac{1}{\left[M^{2}-\left(\vec{k}_{\perp}^{2}+m^{2}\right) / x-\left(\vec{k}_{\perp}^{2}+\lambda^{2}\right) /(1-x)\right]} \\
= & \frac{M e^{2}}{4 \pi^{2}} \int_{0}^{1} d \alpha \int_{0}^{1} d x \frac{x(m-x M)}{\alpha(1-\alpha) \frac{1-x}{x} \vec{q}_{\perp}^{2}-M^{2}+\frac{m^{2}}{x}+\frac{\lambda^{2}}{1-x}}
\end{aligned}
$$

and

$$
\begin{aligned}
B_{\mathrm{b}}\left(q^{2}\right)= & \frac{-2 M}{\left(q^{1}-\mathrm{i} q^{2}\right)}\left\langle\Psi^{\uparrow}\left(P^{+}, \vec{P}_{\perp}=\vec{q}_{\perp}\right)\left|\frac{T_{\mathrm{b}}^{++}(0)}{2\left(P^{+}\right)^{2}}\right| \Psi^{\downarrow}\left(P^{+}, \vec{P}_{\perp}=\overrightarrow{0}_{\perp}\right)\right\rangle \\
= & \frac{-2 M}{\left(q^{1}-\mathrm{i} q^{2}\right)} \int \frac{\mathrm{d}^{2} \vec{k}_{\perp} \mathrm{d} x}{16 \pi^{3}}(1-x) \\
& \times\left[\psi_{+\frac{1}{2}-1}^{\uparrow}\left(x, \vec{k}_{\perp}^{\prime}\right) \psi_{+\frac{1}{2}-1}^{\downarrow}\left(x, \vec{k}_{\perp}\right)+\psi_{-\frac{1}{2}+1}^{\uparrow}\left(x, \vec{k}_{\perp}^{\prime}\right) \psi_{-\frac{1}{2}+1}^{\downarrow}\left(x, \vec{k}_{\perp}\right)\right] \\
= & -4 M \int \frac{\mathrm{d}^{2} \vec{k}_{\perp} \mathrm{d} x}{16 \pi^{3}}(m-M x) \varphi\left(x, \vec{k}_{\perp}^{\prime}\right)^{*} \varphi\left(x, \vec{k}_{\perp}\right) \\
= & -4 M e^{2} \int \frac{\mathrm{d}^{2} \vec{k}_{\perp} \mathrm{d} x}{16 \pi^{3}} \frac{(m-x M)}{(1-x)} \\
& \times \frac{1}{\left[M^{2}-\left(\left(\vec{k}_{\perp}-x \vec{q}_{\perp}\right)^{2}+m^{2}\right) / x-\left(\left(\vec{k}_{\perp}-x \vec{q}_{\perp}\right)^{2}+\lambda^{2}\right) /(1-x)\right]} \\
& \times \frac{1}{\left[M^{2}-\left(\vec{k}_{\perp}^{2}+m^{2}\right) / x-\left(\vec{k}_{\perp}^{2}+\lambda^{2}\right) /(1-x)\right]} \\
= & -\frac{M^{2}}{4 \pi^{2}} \int_{0}^{1} d \alpha \int_{0}^{1} d x \frac{x(m-x M)}{\alpha(1-\alpha) \frac{x}{1-x} \vec{q}_{\perp}^{2}-M^{2}+\frac{m^{2}}{x}+\frac{\lambda^{2}}{1-x}} .
\end{aligned}
$$

The total contribution for general momentum transfer is

$$
\begin{aligned}
& B\left(q^{2}\right)=B_{\mathrm{f}}\left(q^{2}\right)+B_{\mathrm{b}}\left(q^{2}\right) \\
= & 4 M e^{2} \int \frac{\mathrm{d}^{2} \vec{k}_{\perp} \mathrm{d} x}{16 \pi^{3}} \frac{(m-x M)}{(1-x)} \\
\times & \left\{\frac{1}{\left[M^{2}-\left(\left(\vec{k}_{\perp}+(1-x) \vec{q}_{\perp}\right)^{2}+m^{2}\right) / x-\left(\left(\vec{k}_{\perp}+(1-x) \vec{q}_{\perp}\right)^{2}+\lambda^{2}\right) /(1-x)\right]}\right. \\
& \left.\quad-\frac{1}{\left[M^{2}-\left(\left(\vec{k}_{\perp}-x \vec{q}_{\perp}\right)^{2}+m^{2}\right) / x-\left(\left(\vec{k}_{\perp}-x \vec{q}_{\perp}\right)^{2}+\lambda^{2}\right) /(1-x)\right]}\right\} \\
\times & \frac{1}{\left[M^{2}-\left(\vec{k}_{\perp}^{2}+m^{2}\right) / x-\left(\vec{k}_{\perp}^{2}+\lambda^{2}\right) /(1-x)\right]}
\end{aligned}
$$




$$
\begin{aligned}
= & \frac{M e^{2}}{4 \pi^{2}} \int_{0}^{1} d \alpha \int_{0}^{1} d x x(m-x M) \\
& \times\left(\frac{1}{\alpha(1-\alpha) \frac{1-x}{x} \vec{q}_{\perp}^{2}-M^{2}+\frac{m^{2}}{x}+\frac{\lambda^{2}}{1-x}}-\frac{1}{\alpha(1-\alpha) \frac{x}{1-x} \vec{q}_{\perp}^{2}-M^{2}+\frac{m^{2}}{x}+\frac{\lambda^{2}}{1-x}}\right) .
\end{aligned}
$$

This is the analog of the Pauli form factor for a physical electron scattering in a gravitational field and in general is not zero. However at zero momentum transfer

$$
B(0)=B_{\mathrm{f}}(0)+B_{\mathrm{b}}(0)=0,
$$

in agreement with classical arguments based on the equivalence principle and conservation of the energy momentum tensor. [9, 18, 19, 20]

The helicity-flip electromagnetic and gravitational form factors for the fluctuations of the electron at one-loop are illustrated in Fig. 1. The cancellation of the sum of graviton couplings $B\left(q^{2}\right)$ to the constituents at $q^{2}=0$ is evident.

(a) Helicity-flip Pauli form factor $F_{2}\left(q^{2}\right)$ in QED. Notice that $F_{2}(0)=1 / 2$.

(b) Helicity-flip form factor $B_{b}\left(q^{2}\right)$ of the graviton coupling to the boson (photon) constituent of the electron at one-loop order in QED. Notice that $B_{b}(0)=-1 / 3$.

(c) Helicity-flip fermion form factor $B_{f}\left(q^{2}\right)$ of the graviton coupling to the fermion constituent at one-loop order in QED. Notice that $B_{f}(0)=1 / 3$, and thus $B_{f}(0)+$ $B_{b}(0)=0$.

(d) Helicity-flip Pauli form factor $F_{2}\left(q^{2}\right)$ in the Yukawa theory. Notice that in this case $F_{2}(0)=3 / 4$.

(e) Helicity-flip form factor $B_{b}\left(q^{2}\right)$ of the graviton coupling to the boson at oneloop order in the Yukawa theory. Notice that $B_{b}(0)=-5 / 12$.

(f) Helicity-flip fermion form factor $B_{f}\left(q^{2}\right)$ of the graviton coupling to the fermion constituent at one-loop order in the Yukawa theory. Notice that $B_{f}(0)=5 / 12$, and thus $B_{f}(0)+B_{b}(0)=0$.

Figure 1: Helicity-flip electromagnetic and gravitational form factors for spacelike $q^{2}=-Q^{2}<0$ from the quantum fluctuations of a fermion at one-loop order in units of $\alpha / \pi$ for QED and $g^{2} / 4 \pi^{2}$ for the Yukawa theory. The fermion constituent mass is taken as $m_{f}=M$. The boson constituent is massless. 

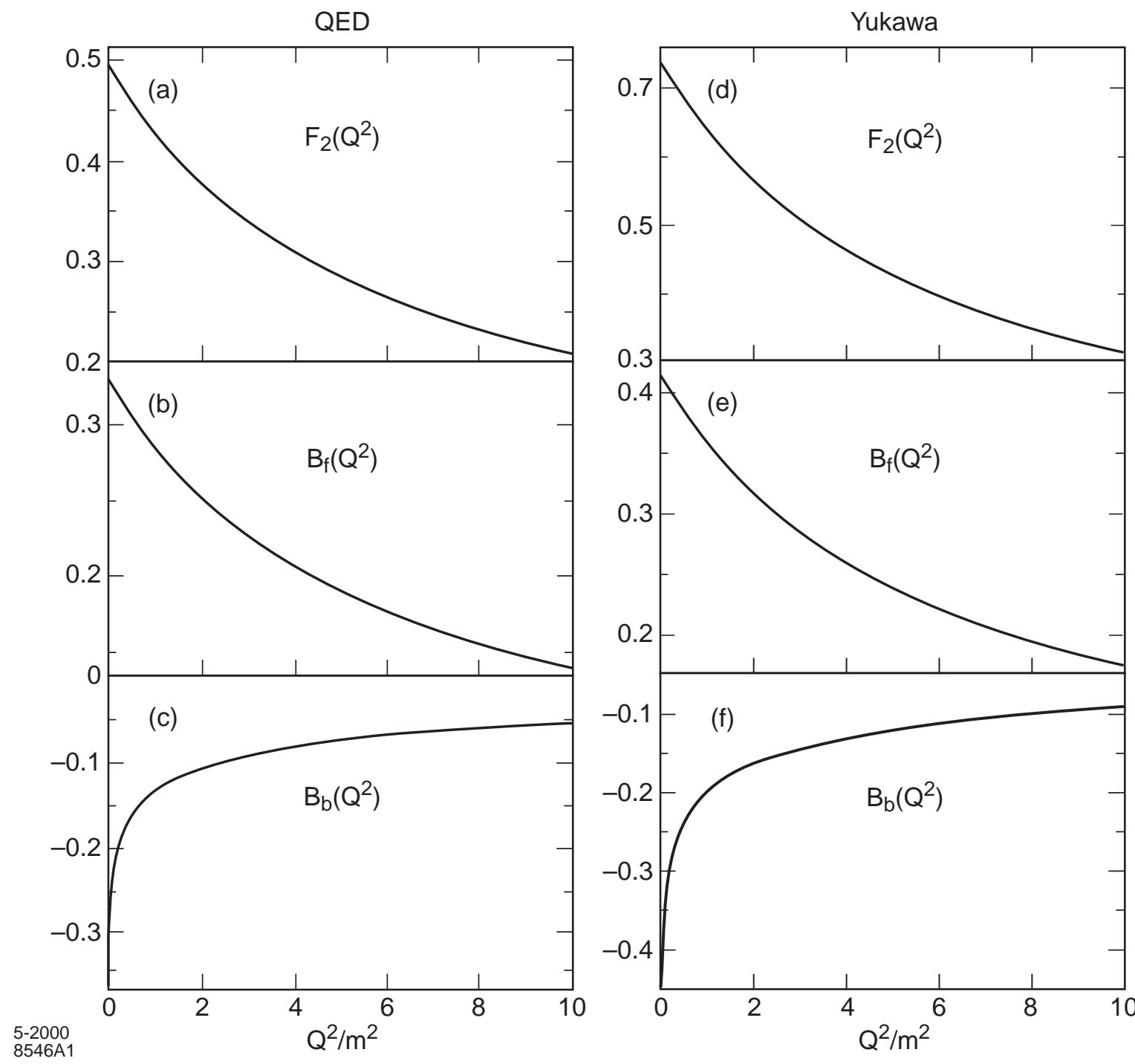
In section 5 we shall prove that the anomalous gravitomagnetic moment $B(0)=$ $B_{\mathrm{f}}(0)+B_{\mathrm{b}}(0)$ is identically zero for arbitrary composite systems in quantum field theory. The proof follows from the Lorentz covariance of the light-cone wavefunction and applies to the contribution of each individual Fock component.

\section{The Light-Cone Fock State Decomposition and Spin Structure of Composite Fermions in Yukawa Theory}

As a second example, we shall consider an effectively composite system composed of a fermion and a neutral scalar based on the one-loop quantum fluctuations of this theory. The light-cone wavefunctions describe off-shell particles but are computable explicitly from perturbation theory. We consider the Yukawa Lagrangian

$$
\begin{aligned}
\mathcal{L} & =\frac{i}{2}\left[\bar{\psi} \gamma^{\mu}\left(\partial_{\mu} \psi\right)-\left(\partial_{\mu} \bar{\psi}\right) \gamma^{\mu} \psi\right]-m \bar{\psi} \psi \\
& +\frac{1}{2}\left(\partial^{\mu} \phi\right)\left(\partial_{\mu} \phi\right)-\frac{1}{2} \lambda^{2} \phi \phi+g \phi \bar{\psi} \psi
\end{aligned}
$$

and the corresponding energy-momentum tensor is given by

$$
T^{\mu \nu}=\frac{i}{2}\left[\bar{\psi} \gamma^{\mu} \vec{\partial}^{\nu} \psi-\bar{\psi} \gamma^{\mu} \overleftarrow{\partial}^{\nu} \psi\right]+\left(\partial^{\mu} \phi\right)\left(\partial^{\nu} \phi\right)-g^{\mu \nu} \mathcal{L},
$$

which is conserved.

The $J^{z}=+\frac{1}{2}$ two-particle Fock state is given by

$$
\begin{aligned}
& \left|\Psi_{\text {two particle }}^{\uparrow}\left(P^{+}, \vec{P}_{\perp}=\overrightarrow{0}_{\perp}\right)\right\rangle \\
= & \int \frac{\mathrm{d}^{2} \vec{k}_{\perp} \mathrm{d} x}{\sqrt{x(1-x)} 16 \pi^{3}}\left[\psi_{+\frac{1}{2}}^{\uparrow}\left(x, \vec{k}_{\perp}\right)\left|+\frac{1}{2} ; x P^{+}, \vec{k}_{\perp}\right\rangle+\psi_{-\frac{1}{2}}^{\uparrow}\left(x, \vec{k}_{\perp}\right)\left|-\frac{1}{2} ; x P^{+}, \vec{k}_{\perp}\right\rangle\right]
\end{aligned}
$$

where

$$
\left\{\begin{array}{l}
\psi_{+\frac{1}{2}}^{\uparrow}\left(x, \vec{k}_{\perp}\right)=\left(M+\frac{m}{x}\right) \varphi, \\
\psi_{-\frac{1}{2}}^{\uparrow}\left(x, \vec{k}_{\perp}\right)=-\frac{\left(+k^{1}+\mathrm{i} k^{2}\right)}{x} \varphi
\end{array}\right.
$$


The scalar part of the wavefunction $\varphi$ is given in Eq. (22) with $e$ replaced by $g$. The normalization of the Fock states is as in (2).

Similarly, the $J^{z}=-\frac{1}{2}$ two-particle Fock state is given by

$$
\begin{aligned}
& \left|\Psi_{\text {two particle }}^{\downarrow}\left(P^{+}, \vec{P}_{\perp}=\overrightarrow{0}_{\perp}\right)\right\rangle \\
= & \int \frac{\mathrm{d}^{2} \vec{k}_{\perp} \mathrm{d} x}{\sqrt{x(1-x)} 16 \pi^{3}}\left[\psi_{+\frac{1}{2}}^{\downarrow}\left(x, \vec{k}_{\perp}\right)\left|+\frac{1}{2} ; x P^{+}, \vec{k}_{\perp}\right\rangle+\psi_{-\frac{1}{2}}^{\downarrow}\left(x, \vec{k}_{\perp}\right)\left|-\frac{1}{2} ; x P^{+}, \vec{k}_{\perp}\right\rangle\right],
\end{aligned}
$$

where

$$
\left\{\begin{array}{l}
\psi_{+\frac{1}{2}}^{\downarrow}\left(x, \vec{k}_{\perp}\right)=\frac{\left(+k^{1}-\mathrm{i} k^{2}\right)}{x} \varphi, \\
\psi_{-\frac{1}{2}}^{\downarrow}\left(x, \vec{k}_{\perp}\right)=\left(M+\frac{m}{x}\right) \varphi .
\end{array}\right.
$$

In (44) and (46) we have generalized the framework of the Yukawa theory by assigning a mass $M$ to the external electrons, but a different mass $m$ to the internal electron lines and a mass $\lambda$ to the internal boson line [8]. The idea behind this is to model the structure of a composite fermion state with mass $M$ by a fermion and a boson constituent with respective masses $m$ and $\lambda$.

Using Eqs. (5) and (43) we have

$$
F_{1}\left(q^{2}\right)=Z+\int \frac{\mathrm{d}^{2} \vec{k}_{\perp} \mathrm{d} x}{16 \pi^{3}}\left[\psi_{+\frac{1}{2}}^{\uparrow *}\left(x, \vec{k}_{\perp}^{\prime}\right) \psi_{+\frac{1}{2}}^{\uparrow}\left(x, \vec{k}_{\perp}\right)+\psi_{-\frac{1}{2}}^{\uparrow *}\left(x, \vec{k}_{\perp}^{\prime}\right) \psi_{-\frac{1}{2}}^{\uparrow}\left(x, \vec{k}_{\perp}\right)\right]
$$

where $\vec{k}_{\perp}^{\prime}$ is given in Eq. (27). At zero momentum transfer

$$
F_{1}(0)=Z+\int \frac{\mathrm{d}^{2} \vec{k}_{\perp} \mathrm{d} x}{16 \pi^{3}}\left[\psi_{+\frac{1}{2}}^{\uparrow *}\left(x, \vec{k}_{\perp}\right) \psi_{+\frac{1}{2}}^{\uparrow}\left(x, \vec{k}_{\perp}\right)+\psi_{-\frac{1}{2}}^{\uparrow *}\left(x, \vec{k}_{\perp}\right) \psi_{-\frac{1}{2}}^{\uparrow}\left(x, \vec{k}_{\perp}\right)\right]
$$

where the renormalization constant $Z$ in light-cone gauge is given, using Pauli-Villars regularization, by

$$
\begin{aligned}
Z=1- & \frac{1}{4 \pi}\left(\frac{g^{2}}{4 \pi}\right) \int_{0}^{1} d x\left[(1-x) \ln \frac{-M^{2}+\frac{m^{2}}{x}+\frac{\Lambda^{2}}{1-x}}{-M^{2}+\frac{m^{2}}{x}+\frac{\lambda^{2}}{1-x}}\right. \\
& \left.+\frac{(x M+m)^{2}}{x}\left(-\frac{1}{-M^{2}+\frac{m^{2}}{x}+\frac{\Lambda^{2}}{1-x}}+\frac{1}{-M^{2}+\frac{m^{2}}{x}+\frac{\lambda^{2}}{1-x}}\right)\right] .
\end{aligned}
$$

The Pauli form factor is obtained from the spin-flip matrix element of the $J^{+}$ current. From Eqs. (5), (43) and (45) we have

$$
F_{2}\left(q^{2}\right)=\frac{-2 M}{\left(q^{1}-\mathrm{i} q^{2}\right)} \int \frac{\mathrm{d}^{2} \vec{k}_{\perp} \mathrm{d} x}{16 \pi^{3}}\left[\psi_{+\frac{1}{2}}^{\uparrow}\left(x, \vec{k}_{\perp}^{\prime}\right) \psi_{+\frac{1}{2}}^{\downarrow}\left(x, \vec{k}_{\perp}\right)+\psi_{-\frac{1}{2}}^{\uparrow *}\left(x, \vec{k}_{\perp}^{\prime}\right) \psi_{-\frac{1}{2}}^{\downarrow}\left(x, \vec{k}_{\perp}\right)\right]
$$




$$
\begin{aligned}
= & 2 M \int \frac{\mathrm{d}^{2} \vec{k}_{\perp} \mathrm{d} x}{16 \pi^{3}} \frac{(1-x)(m+M x)}{x^{2}} \varphi\left(x, \vec{k}_{\perp}^{\prime}\right)^{*} \varphi\left(x, \vec{k}_{\perp}\right) \\
= & 2 M g^{2} \int \frac{\mathrm{d}^{2} \vec{k}_{\perp} \mathrm{d} x}{16 \pi^{3}} \frac{(m+x M)}{x^{2}} \\
& \times \frac{1}{\left[M^{2}-\left(\left(\vec{k}_{\perp}+(1-x) \vec{q}_{\perp}\right)^{2}+m^{2}\right) / x-\left(\left(\vec{k}_{\perp}+(1-x) \vec{q}_{\perp}\right)^{2}+\lambda^{2}\right) /(1-x)\right]} \\
& \times \frac{1}{\left[M^{2}-\left(\vec{k}_{\perp}^{2}+m^{2}\right) / x-\left(\vec{k}_{\perp}^{2}+\lambda^{2}\right) /(1-x)\right]} \\
= & \frac{M g^{2}}{8 \pi^{2}} \int_{0}^{1} d \alpha \int_{0}^{1} d x \frac{\frac{1-x}{x}(m+x M)}{\alpha(1-\alpha) \frac{1-x}{x} \vec{q}_{\perp}^{2}-M^{2}+\frac{m^{2}}{x}+\frac{\lambda^{2}}{1-x}} .
\end{aligned}
$$

The anomalous moment is obtained in the limit of zero momentum transfer,

$$
\begin{aligned}
F_{2}(0) & =2 M g^{2} \int \frac{\mathrm{d}^{2} \vec{k}_{\perp} \mathrm{d} x}{16 \pi^{3}} \frac{(m+x M)}{x^{2}} \frac{1}{\left[M^{2}-\left(\vec{k}_{\perp}^{2}+m^{2}\right) / x-\left(\vec{k}_{\perp}^{2}+\lambda^{2}\right) /(1-x)\right]^{2}} \\
& =\frac{M g^{2}}{8 \pi^{2}} \int_{0}^{1} d x \frac{\frac{1-x}{x}(m+x M)}{-M^{2}+\frac{m^{2}}{x}+\frac{\lambda^{2}}{1-x}} .
\end{aligned}
$$

The individual contributions of the fermion and boson fields to the energy-momentum form factors in the Yukawa model are given by

$$
\begin{aligned}
A_{\mathrm{f}}\left(q^{2}\right) & =\left\langle\Psi^{\uparrow}\left(P^{+}, \vec{P}_{\perp}=\vec{q}_{\perp}\right)|x| \Psi^{\uparrow}\left(P^{+}, \vec{P}_{\perp}=\overrightarrow{0}_{\perp}\right)\right\rangle \\
& =\int \frac{\mathrm{d}^{2} \vec{k}_{\perp} \mathrm{d} x}{16 \pi^{3}} x\left[\psi_{+\frac{1}{2}}^{\uparrow *}\left(x, \vec{k}_{\perp}^{\prime}\right) \psi_{+\frac{1}{2}}^{\uparrow}\left(x, \vec{k}_{\perp}\right)+\psi_{-\frac{1}{2}}^{\uparrow *}\left(x, \vec{k}_{\perp}^{\prime}\right) \psi_{-\frac{1}{2}}^{\uparrow}\left(x, \vec{k}_{\perp}\right)\right]
\end{aligned}
$$

where $\vec{k}_{\perp}^{\prime}=\vec{k}_{\perp}+(1-x) \vec{q}_{\perp}$, and

$$
\begin{aligned}
A_{\mathrm{b}}\left(q^{2}\right) & =\left\langle\Psi^{\uparrow}\left(P^{+}, \vec{P}_{\perp}=\vec{q}_{\perp}\right)|(1-x)| \Psi^{\uparrow}\left(P^{+}, \vec{P}_{\perp}=\overrightarrow{0}_{\perp}\right)\right\rangle \\
& =\int \frac{\mathrm{d}^{2} \vec{k}_{\perp} \mathrm{d} x}{16 \pi^{3}}(1-x)\left[\psi_{+\frac{1}{2}}^{\uparrow}\left(x, \vec{k}_{\perp}^{\prime \prime}\right) \psi_{+\frac{1}{2}}^{\uparrow}\left(x, \vec{k}_{\perp}\right)+\psi_{-\frac{1}{2}}^{\uparrow *}\left(x, \vec{k}_{\perp}^{\prime \prime}\right) \psi_{-\frac{1}{2}}^{\uparrow}\left(x, \vec{k}_{\perp}\right)\right],
\end{aligned}
$$

where in this case $\vec{k}_{\perp}^{\prime \prime}=\vec{k}_{\perp}-x \vec{q}_{\perp}$. Note that again

$$
A_{\mathrm{f}}(0)+A_{\mathrm{b}}(0)=F_{1}(0)=1
$$

which corresponds to the momentum sum rule.

The fermion and boson contributions to the spin-flip matter form factor are

$$
B_{\mathrm{f}}\left(q^{2}\right)=\frac{-2 M}{\left(q^{1}-\mathrm{i} q^{2}\right)} \int \frac{\mathrm{d}^{2} \vec{k}_{\perp} \mathrm{d} x}{16 \pi^{3}} x
$$




$$
\begin{aligned}
& \times\left[\psi_{+\frac{1}{2}}^{\uparrow *}\left(x, \vec{k}_{\perp}^{\prime}\right) \psi_{+\frac{1}{2}}^{\downarrow}\left(x, \vec{k}_{\perp}\right)+\psi_{-\frac{1}{2}}^{\uparrow *}\left(x, \vec{k}_{\perp}^{\prime}\right) \psi_{-\frac{1}{2}}^{\downarrow}\left(x, \vec{k}_{\perp}\right)\right] \\
= & 2 M \int \frac{\mathrm{d}^{2} \vec{k}_{\perp} \mathrm{d} x}{16 \pi^{3}} \frac{(1-x)(m+M x)}{x} \varphi\left(x, \vec{k}_{\perp}^{\prime}\right)^{*} \varphi\left(x, \vec{k}_{\perp}\right) \\
= & 2 M g^{2} \int \frac{\mathrm{d}^{2} \vec{k}_{\perp} \mathrm{d} x}{16 \pi^{3}} \frac{1}{x} \\
& \times \frac{1}{\left[M^{2}-\left(\left(\vec{k}_{\perp}+(1-x) \vec{q}_{\perp}\right)^{2}+m^{2}\right) / x-\left(\left(\vec{k}_{\perp}+(1-x) \vec{q}_{\perp}\right)^{2}+\lambda^{2}\right) /(1-x)\right]} \\
& \times \frac{1}{\left[M^{2}-\left(\vec{k}_{\perp}^{2}+m^{2}\right) / x-\left(\vec{k}_{\perp}^{2}+\lambda^{2}\right) /(1-x)\right]} \\
= & \frac{M g^{2}}{8 \pi^{2}} \int_{0}^{1} d \alpha \int_{0}^{1} d x \frac{(1-x)(m+x M)}{\alpha(1-\alpha) \frac{1-x}{x} \vec{q}_{\perp}^{2}-M^{2}+\frac{m^{2}}{x}+\frac{\lambda^{2}}{1-x}} .
\end{aligned}
$$

and

$$
\begin{aligned}
B_{\mathrm{b}}\left(q^{2}\right)= & \frac{-2 M}{\left(q^{1}-\mathrm{i} q^{2}\right)} \int \frac{\mathrm{d}^{2} \vec{k}_{\perp} \mathrm{d} x}{16 \pi^{3}}(1-x) \\
& \times\left[\psi_{+\frac{1}{2}-1}^{\uparrow *}\left(x, \vec{k}_{\perp}^{\prime}\right) \psi_{+\frac{1}{2}-1}^{\downarrow}\left(x, \vec{k}_{\perp}\right)+\psi_{-\frac{1}{2}+1}^{\uparrow *}\left(x, \vec{k}_{\perp}^{\prime}\right) \psi_{-\frac{1}{2}+1}^{\downarrow}\left(x, \vec{k}_{\perp}\right)\right] \\
= & -2 M \int \frac{\mathrm{d}^{2} \vec{k}_{\perp} \mathrm{d} x}{16 \pi^{3}} \frac{(1-x)(m+M x)}{x} \varphi\left(x, \vec{k}_{\perp}^{\prime \prime}\right)^{*} \varphi\left(x, \vec{k}_{\perp}\right) \\
= & -2 M g^{2} \int \frac{\mathrm{d}^{2} \vec{k}_{\perp} \mathrm{d} x}{16 \pi^{3}} \frac{(m+x M)}{x} \\
& \times \frac{1}{\left[M^{2}-\left(\left(\vec{k}_{\perp}-x \vec{q}_{\perp}\right)^{2}+m^{2}\right) / x-\left(\left(\vec{k}_{\perp}-x \vec{q}_{\perp}\right)^{2}+\lambda^{2}\right) /(1-x)\right]} \\
& \times \frac{1}{\left[M^{2}-\left(\vec{k}_{\perp}^{2}+m^{2}\right) / x-\left(\vec{k}_{\perp}^{2}+\lambda^{2}\right) /(1-x)\right]} \\
= & -\frac{M^{2}}{8 \pi^{2}} \int_{0}^{1} d \alpha \int_{0}^{1} d x \frac{(1-x)\left(m+x M^{2}\right)}{\alpha(1-\alpha) \frac{x}{1-x} \vec{q}_{\perp}^{2}-M^{2}+\frac{m^{2}}{x}+\frac{\lambda^{2}}{1-x}}
\end{aligned}
$$

The total contribution from the fermion and boson constituents is

$$
\begin{aligned}
& B\left(q^{2}\right)=B_{\mathrm{f}}\left(q^{2}\right)+B_{\mathrm{b}}\left(q^{2}\right) \\
= & 2 M g^{2} \int \frac{\mathrm{d}^{2} \vec{k}_{\perp} \mathrm{d} x}{16 \pi^{3}} \frac{(m+x M)}{x} \\
& \times\left\{\frac{1}{\left[M^{2}-\left(\left(\vec{k}_{\perp}+(1-x) \vec{q}_{\perp}\right)^{2}+m^{2}\right) / x-\left(\left(\vec{k}_{\perp}+(1-x) \vec{q}_{\perp}\right)^{2}+\lambda^{2}\right) /(1-x)\right]}\right. \\
& \left.\quad-\frac{1}{\left[M^{2}-\left(\left(\vec{k}_{\perp}-x \vec{q}_{\perp}\right)^{2}+m^{2}\right) / x-\left(\left(\vec{k}_{\perp}-x \vec{q}_{\perp}\right)^{2}+\lambda^{2}\right) /(1-x)\right]}\right\} \\
& \times \frac{1}{\left[M^{2}-\left(\vec{k}_{\perp}^{2}+m^{2}\right) / x-\left(\vec{k}_{\perp}^{2}+\lambda^{2}\right) /(1-x)\right]}
\end{aligned}
$$




$$
\begin{aligned}
= & \frac{M g^{2}}{8 \pi^{2}} \int_{0}^{1} d \alpha \int_{0}^{1} d x(1-x)(m+x M) \\
& \times\left(\frac{1}{\alpha(1-\alpha) \frac{1-x}{x} \vec{q}_{\perp}^{2}-M^{2}+\frac{m^{2}}{x}+\frac{\lambda^{2}}{1-x}}-\frac{1}{\alpha(1-\alpha) \frac{x}{1-x} \vec{q}_{\perp}^{2}-M^{2}+\frac{m^{2}}{x}+\frac{\lambda^{2}}{1-x}}\right) .
\end{aligned}
$$

At zero momentum transfer

$$
B(0)=B_{\mathrm{f}}(0)+B_{\mathrm{b}}(0)=0,
$$

which is another example of the vanishing of the anomalous gravitomagnetic moment. [See also Fig. 1(e) + Fig. 1(f).] The general proof that $B(0)=0$ for any system is given in the next section. Note that $B\left(Q^{2}\right)$ does not vanish for nonzero momentum transfer.

\section{The Anomalous Gravitomagnetic Moment for Composite Systems}

In this section we shall show that the anomalous gravitomagnetic moment $B(0)$ always vanishes for each contributing Fock state of a general composite system. In order to calculate $B(0)$ using Eq. (14), we need to consider a non-forward amplitude. The internal momentum variables for the final state wavefunction are given by Eqs. (10)

and (11). The subscripts of $x_{i}$ and $\vec{k}_{\perp i}$ label constituent particles, the superscripts of $q_{\perp}^{1}, k_{\perp}^{1}$, and $k_{\perp}^{2}$ label the Lorentz indices, and the subscript $a$ in $\psi_{a}$ indicates the contributing Fock state. The essential ingredient is the Lorentz property of the light-cone wavefunctions.

It is important to identify the $n-1$ independent relative momenta of the $n$-particle Fock state.

$$
\begin{aligned}
& -\frac{B(0)}{2 M}=\lim _{q_{\perp}^{1} \rightarrow 0} \frac{\partial}{\partial q_{\perp}^{1}}\left\langle P+q, \uparrow\left|\frac{T^{++}(0)}{2\left(P^{+}\right)^{2}}\right| P, \downarrow\right\rangle \\
= & \left.\lim _{q_{\perp}^{1} \rightarrow 0} \frac{\partial}{\partial q_{\perp}^{1}}\left\langle\Psi^{\uparrow}\left(P^{+}=1, \vec{P}_{\perp}=\vec{q}_{\perp}\right)\right)\left|\frac{T^{++}(0)}{2\left(P^{+}\right)^{2}}\right| \Psi^{\downarrow}\left(P^{+}=1, \vec{P}_{\perp}=\overrightarrow{0}_{\perp}\right)\right\rangle \\
= & \lim _{q_{\perp}^{1} \rightarrow 0} \frac{\partial}{\partial q_{\perp}^{1}} \sum_{a} \int \prod_{k=1}^{n-1} \frac{\mathrm{d}^{2} \vec{k}_{\perp k} \mathrm{~d} x_{k}}{16 \pi^{3}} \psi_{a}^{\uparrow *}\left(x_{1}, x_{2}, \cdots, x_{n-1},\left(1-x_{1}-x_{2}-\cdots-x_{n-1}\right),\right.
\end{aligned}
$$




$$
\begin{gathered}
\left.\vec{k}_{\perp 1}^{\prime}, \vec{k}_{\perp 2}^{\prime}, \cdots, \vec{k}_{\perp n-1}^{\prime},\left(-\vec{k}_{\perp 1}^{\prime}-\vec{k}_{\perp 2}^{\prime}-\cdots-\vec{k}_{\perp n-1}^{\prime}\right)\right) \\
\times \quad\left[\sum_{i=1}^{n-1} x_{i}+\left(1-x_{1}-x_{2}-\cdots-x_{n-1}\right)\right] \\
\times \quad \psi_{a}^{\downarrow}\left(x_{1}, x_{2}, \cdots, x_{n-1},\left(1-x_{1}-x_{2}-\cdots-x_{n-1}\right),\right. \\
\left.\vec{k}_{\perp 1}, \vec{k}_{\perp 2}, \cdots, \vec{k}_{\perp n-1},\left(-\vec{k}_{\perp 1}-\vec{k}_{\perp 2}-\cdots-\vec{k}_{\perp n-1}\right)\right) .
\end{gathered}
$$

Using integration by parts,

$$
\begin{aligned}
&-\frac{B_{a}(0)}{2 M}= \\
&= \int \prod_{k=1}^{n-1} \frac{\mathrm{d}^{2} \vec{k}_{\perp k} \mathrm{~d} x_{k}}{16 \pi^{3}} \psi_{a}^{\uparrow *}\left(x_{1}, x_{2}, \cdots, x_{n-1},\left(1-x_{1}-x_{2}-\cdots-x_{n-1}\right),\right. \\
&\left.\vec{k}_{\perp 1}, \vec{k}_{\perp 2}, \cdots, \vec{k}_{\perp n-1},\left(-\vec{k}_{\perp 1}-\vec{k}_{\perp 2}-\cdots-\vec{k}_{\perp n-1}\right)\right) \\
& \times {\left[\sum_{i=1}^{n-1} x_{i}\left(\left(-1+x_{i}\right) \frac{\partial}{\partial k_{\perp i}^{1}}+\sum_{j \neq i}^{n-1} x_{j} \frac{\partial}{\partial k_{\perp j}^{1}}\right)+\left(1-x_{1}-x_{2}-\cdots-x_{n-1}\right) \sum_{j=1}^{n-1} x_{j} \frac{\partial}{\partial k_{\perp j}^{1}}\right] } \\
& \times \psi_{a}^{\downarrow}\left(x_{1}, x_{2}, \cdots, x_{n-1},\left(1-x_{1}-x_{2}-\cdots-x_{n-1}\right),\right. \\
&= \prod_{k=1}^{n-1} \frac{\mathrm{d}^{2} \vec{k}_{\perp k} \mathrm{~d} x_{k}}{16 \pi^{3}} \psi_{a}^{\uparrow *}\left(x_{1}, x_{2}, \cdots, x_{n-1},\left(1-x_{1}-x_{2}-\cdots-x_{n-1}\right),\right. \\
&\left.\vec{k}_{\perp 1}, \vec{k}_{\perp 2}, \cdots, \vec{k}_{\perp n-1},\left(-\vec{k}_{\perp 1}-\vec{k}_{\perp 2}-\cdots-\vec{k}_{\perp n-1}\right)\right) \\
& \times \quad\left[\sum_{i=1}^{n-1}\left(-1+\sum_{j=1}^{n-1} x_{j}+\left(1-x_{1}-x_{2}-\cdots-x_{n-1}\right)\right) x_{i} \frac{\partial}{\partial k_{\perp i}^{1}}\right] \\
& \times \quad \psi_{a}^{\downarrow}\left(x_{1}, x_{2}, \cdots, x_{n-1},\left(1-x_{1}-x_{2}-\cdots-x_{n-1}\right),\right. \\
&\left.\vec{k}_{\perp 1}, \vec{k}_{\perp 2}, \cdots, \vec{k}_{\perp n-1},\left(-\vec{k}_{\perp 1}-\vec{k}_{\perp 2}-\cdots-\vec{k}_{\perp n-1}\right)\right) \\
&= 0
\end{aligned}
$$

Thus the contribution $B_{a}(0)$ from each contributing Fock state $a$ to the total anomalous gravitomagnetic moment $B(0)$ vanishes separately. 


\section{The Perturbative Models as a Template for a Composite System}

We can use the structure of the one-loop QED and Yukawa calculations with general values for $M, m$, and $\lambda$, to represent a spin- $\frac{1}{2}$ system composed of a fermion and a spin-1 or spin-0 boson. Such a model describes an effectively composite system with no bare one-particle Fock state. We can also generalize the functional form of the

momentum space wavefunction $\varphi\left(x, \vec{k}_{\perp}\right)$ by introducing a spectrum of vector bosons satisfying the generalized Pauli-Villars spectral conditions

$$
\int d \lambda^{2} \lambda^{2 N} \rho\left(\lambda^{2}\right)=0, \quad N=0,1, \cdots
$$

For example, we can simulate a proton as a bound state of a quark and diquark [24, using spin-0, spin-1 diquarks, or a linear superposition of the two states. The model can be made to match the power-law fall-off of the hadron form factors predicted in perturbative QCD by the choice of sum rule conditions on the Pauli-Villars spectra. [25, 26]. The light-cone framework of the model resembles that of the covariant parton model of Landshoff, Polkinghorne and Short [27, 28], in which the power behavior of the spectral integral at high masses corresponds to the Regge behavior of the deep inelastic structure functions. Although the model is based on just two Fock constituents, it is relativistic and satisfies self-consistency conditions such as in the point-like limit where $R^{2} M^{2} \rightarrow 0$, the anomalous moment vanishes. [22] The light-cone formalism also properly incorporates Wigner boosts. Thus this model of composite systems can serve as a useful theoretical laboratory to interrelate hadronic properties and check the consistency of formulae proposed for the study of hadron substructure. 


\section{$7 \quad$ Spin and Orbital Angular Momentum Compo- sition of Light-Cone Wavefunctions}

In general the light-cone wavefunctions satisfy conservation of the $z$ projection of angular momentum:

$$
J^{z}=\sum_{i=1}^{n} s_{i}^{z}+\sum_{j=1}^{n-1} l_{j}^{z} .
$$

The sum over $s_{i}^{z}$ represents the contribution of the intrinsic spins of the $n$ Fock state constituents. The sum over orbital angular momenta $l_{j}^{z}=-\mathrm{i}\left(k_{j}^{1} \frac{\partial}{\partial k_{j}^{2}}-k_{j}^{2} \frac{\partial}{\partial k_{j}^{1}}\right)$ derives from the $n-1$ relative momenta. This excludes the contribution to the orbital angular momentum due to the motion of the center of mass, which is not an intrinsic property of the hadron.

We can see how the angular momentum sum rule Eq. (62) is satisfied for the wavefunctions Eqs. (20) and (23) of the QED model system of two-particle Fock states. In Table 1 we list the fermion constituent's light-cone spin projection $s_{\mathrm{f}}^{z}=\frac{1}{2} \lambda_{\mathrm{f}}$, the boson constituent spin projection $s_{\mathrm{b}}^{z}=\lambda_{\mathrm{b}}$, and the relative orbital angular momentum $l^{z}$ for each contributing configuration of the QED model system wavefunction. Table 1 is derived by calculating the matrix elements of the light-cone helicity opera-

Table 1. Spin Decomposition of the $J_{e}^{z}=+1 / 2$ Electron

\begin{tabular}{|c|c|c|c|}
\hline Configuration & Fermion Spin $s_{\mathrm{f}}^{z}$ & Boson Spin $s_{\mathrm{b}}^{z}$ & Orbital Ang. Mom. $l^{z}$ \\
\hline \hline$\left|+\frac{1}{2}\right\rangle \rightarrow\left|+\frac{1}{2}+1\right\rangle$ & $+\frac{1}{2}$ & +1 & -1 \\
\hline$\left|+\frac{1}{2}\right\rangle \rightarrow\left|-\frac{1}{2}+1\right\rangle$ & $-\frac{1}{2}$ & +1 & 0 \\
\hline$\left|+\frac{1}{2}\right\rangle \rightarrow\left|+\frac{1}{2}-1\right\rangle$ & $+\frac{1}{2}$ & -1 & +1 \\
\hline
\end{tabular}

tor $\gamma^{+} \gamma^{5}$ [29] and the relative orbital angular momentum operator $-\mathrm{i}\left(k^{1} \frac{\partial}{\partial k^{2}}-k^{2} \frac{\partial}{\partial k^{1}}\right)$ [16, 30, 31 in the light-cone representation. Each configuration satisfies the spin sum 
rule: $J^{z}=s_{\mathrm{f}}^{z}+s_{\mathrm{b}}^{z}+l^{z}$.

For a better understanding of Table 1, we look at the non-relativistic and ultrarelativistic limits. At the non-relativistic limit, the transversal motions of the constituent can be neglected and we have only the $\left|+\frac{1}{2}\right\rangle \rightarrow\left|-\frac{1}{2}+1\right\rangle$ configuration which is the non-relativistic quantum state for the spin-half system composed of a fermion and a spin-1 boson constituents. The fermion constituent has spin projection in the opposite direction to the spin $J^{z}$ of the whole system. However, for ultra-relativistic binding in which the transversal motions of the constituents are large compared to the fermion masses, the $\left|+\frac{1}{2}\right\rangle \rightarrow\left|+\frac{1}{2}+1\right\rangle$ and $\left|+\frac{1}{2}\right\rangle \rightarrow\left|+\frac{1}{2}-1\right\rangle$ configurations dominate over the $\left|+\frac{1}{2}\right\rangle \rightarrow\left|-\frac{1}{2}+1\right\rangle$ configuration. In this case the fermion constituent has spin projection parallel to $J^{z}$.

Table 2. Spin Decomposition of the $J^{z}=+1 / 2$ Fermion in Yukawa Theory

\begin{tabular}{|c|c|c|c|}
\hline Configuration & Fermion Spin $s_{\mathrm{f}}^{z}$ & Boson Spin $s_{\mathrm{b}}^{z}$ & Orbital Ang. Mom. $l^{z}$ \\
\hline \hline$\left|+\frac{1}{2}\right\rangle \rightarrow\left|+\frac{1}{2}\right\rangle$ & $+\frac{1}{2}$ & 0 & 0 \\
\hline$\left|+\frac{1}{2}\right\rangle \rightarrow\left|-\frac{1}{2}\right\rangle$ & $-\frac{1}{2}$ & 0 & +1 \\
\hline
\end{tabular}

The corresponding spin content in the Yukawa theory is given in Table 2. In this case, the non-relativistic fermion's spin projection is aligned with the total $J^{z}$, and it is anti-aligned in the ultra-relativistic limit. The distinct features of spin structure in the non-relativistic and ultra-relativistic limits reveals the importance of relativistic effects and supports the viewpoint [29, 32, 33] that the proton "spin puzzle" can be understood as due to the relativistic motion of quarks inside the nucleon. In particular, the spin projection of the relativistic constituent quark tends to be antialigned with the proton spin in a quark-diquark bound state if the diquark has spin 0 . The state with orbital angular momentum $l^{z}= \pm 1$ in fact dominates over the states with $l^{z}=0$. Thus the empirical fact that $\Delta q$ is small in the proton has a natural 
description in the light-cone Fock representation of hadrons.

The explicit formulas for the quark spin distributions $\Delta q\left(x, \Lambda^{2}\right)$ in the quarkdiquark models can be immediately obtained for the spin-1 diquark model from Eqs. (20) and (21):

$$
\begin{aligned}
& \Delta q\left(x, \Lambda^{2}\right)_{\text {spin-1 diquark }} \\
= & \int \frac{\mathrm{d}^{2} \vec{k}_{\perp} \mathrm{d} x}{16 \pi^{3}} \theta\left(\Lambda^{2}-\mathcal{M}^{2}\right) 2\left[\frac{\vec{k}_{\perp}^{2}}{x^{2}(1-x)^{2}}+\frac{\vec{k}_{\perp}^{2}}{(1-x)^{2}}-\left(M-\frac{m}{x}\right)^{2}\right]|\varphi|^{2},
\end{aligned}
$$

and for the spin-0 diquark model from Eqs. (43) and (44):

$$
\Delta q\left(x, \Lambda^{2}\right)_{\text {spin-0 diquark }}=\int \frac{\mathrm{d}^{2} \vec{k}_{\perp} \mathrm{d} x}{16 \pi^{3}} \theta\left(\Lambda^{2}-\mathcal{M}^{2}\right)\left[\left(M+\frac{m}{x}\right)^{2}-\frac{\vec{k}_{\perp}^{2}}{x^{2}}\right]|\varphi|^{2},
$$

where we have regulated the integral by assuming a cutoff in the invariant mass:

$\mathcal{M}^{2}=\sum_{i} \frac{\vec{k}_{\perp i}^{2}+m_{i}^{2}}{x_{i}}<\Lambda^{2}$. Again, one sees the transition of $\Delta q$ from the nonrelativistic to relativistic limit. In the spin-0 diquark model $\Delta q=1$ in the nonrelativistic limit, and decreases toward $\Delta q=-1$ as the intrinsic transverse momentum increases. The behavior is just opposite in the case of the spin-1 diquark.

\section{Conclusions}

The LC wavefunctions $\psi_{n / H}\left(x_{i}, \vec{k}_{\perp i}, \lambda_{i}\right)$ provide a general representation of a relativistic composite system. They are universal, process independent, and control all hadronic reactions. In this paper we have constructed explicit models which are simple but yet are completely relativistic, preserve all of the Lorentz properties of a composite system of quantum field theory. Because of this explicit realization we can see how different hadronic phenomena can be interrelated. For example, the matrix elements of local operators such as the electromagnetic current, the energy momentum tensor, angular momentum, and the moments of structure functions have exact representations in terms of light-cone Fock state wavefunctions of bound states such as hadrons. We have shown that each Fock state of a composite system satisfies $J_{z}$ conservation, component by component. We have emphasized that the correct 
expression for the orbital angular momentum $l^{z}$ involves a sum over $n-1$ relative momentum contributions for a Fock state with $n$ constituents.

We have illustrated these properties by examining the explicit form of the lightcone wavefunctions for the two-particle Fock state of the electron in QED, thus connecting the Schwinger anomalous magnetic moment to the spin and orbital momentum carried by its Fock state constituents. We have also computed the QED one-loop radiative corrections for the form factors for the graviton coupling to the electron and photon. The one-loop model provides a transparent basis for understanding the structure of relativistic composite systems and their matrix elements in hadronic physics. Although the underlying model is derived from elementary QED perturbative couplings, it in fact can be used to model much more general bound state systems by applying spectral integration over the constituent masses while preserving all of the Lorentz properties.

We thus have obtained a theoretical laboratory to test the consistency of formulae which have been proposed to probe the spin structure of hadrons. For example, we have computed the quark spin distributions $\Delta q\left(x, \Lambda^{2}\right)$ in quark-diquark models. In particular, the spin projection of the relativistic constituent quark tends to be antialigned with the proton spin in a quark-diquark bound state if the diquark has spin 0. The empirical fact that $\Delta q$ is small in the proton thus has a natural description in the light-cone Fock representation of a relativistic bound state.

We have also given general exact expressions for the matrix elements of the electromagnetic, electroweak, and graviton couplings for operators for arbitrary composite systems, giving explicit realization of the spin sum rules and other local matrix elements in terms of the light-cone Fock state wavefunctions.

Finally, we have given a general proof demonstrating that the anomalous gravitomagnetic moment $B(0)$ for gravitons coupling to matter vanishes identically for any composite system. At one loop order in QED, we can see the explicit cancellation of the graviton coupling to the lepton and photon. In fact we have shown that this remarkable property holds generally for any composite or elementary system at all 
orders directly from the Lorentz boost properties of the light-cone Fock representation.

\section{References}

[1] S. D. Drell and T. M. Yan, Phys. Rev. Lett. 24 (1970) 181.

[2] G. B. West, Phys. Rev. Lett. 24 (1970) 1206.

[3] For a review and further references see S. J. Brodsky, H. Pauli, and S. S. Pinsky, Phys. Rept. 301 (1998) 299.

[4] P.A.M. Dirac, Rev. Mod. Phys. 21 (1949) 392.

[5] The notation and calculational rules can be found in: G. P. Lepage and S. J. Brodsky, Phys. Rev. D 22 (1980) 2157; Phys. Lett. B 87 (1979) 359; Phys. Rev. Lett. 43 (1979) 545, 1625(E).

[6] B. Geyer, D. Robaschik, M. Bordag, and J. Hořejší, Z. Phys. C 26 (1985) 591; T. Braunschweig, B. Geyer, J. Hořejší, and D. Robaschik, Z. Phys. C 33 (1987) 275; F.-M. Dittes, D. Müller, D. Robaschik, and B. Geyer, Phys. Lett. B 209 (1988) 325; I. I. Balitskii and V. M. Braun, Nucl. Phys. B 311 (1988/89) 541; P. Jain and J. P. Ralston, in Future Directions in Particle and Nuclear Physics at Multi-GeV Hadron Beam Facilities, BNL, March 1993.

[7] S. J. Brodsky, L. Frankfurt, J. F. Gunion, A. H. Mueller, and M. Strikman, Phys. Rev. D 50 (1994) 3134.

[8] S. J. Brodsky and S. D. Drell, Phys. Rev. D 22 (1980) 2236.

[9] X. Ji, Phys. Rev. D58, 056003 (1998) hep-ph/9710290; X. Ji, Talk presented at 12th Int. Symp. on High-Energy Spin Physics (SPIN96), Amsterdam, Sep. 1996,

hep-ph/9610369; Phys. Rev. Lett. 78 (1997) 610; Phys. Rev. D 55 (1997) 7114. 
[10] S. J. Brodsky and D. S. Hwang, Nucl. Phys. B 543 (1999) 239.

[11] S. J. Chang, R. G. Root and T. M. Yan, Phys. Rev. D 7 (1973) 1133.

[12] M. Burkardt, Nucl. Phys. A 504 (1989) 762; Nucl. Phys. B 373 (1992) 613; Phys. Rev. D 52 (1995) 3841.

[13] H.-M. Choi and C.-R. Ji, Phys. Rev. D 58 (1998) 071901.

[14] S. A. Paston, V. A. Franke and E. V. Prokhvatilov, Theor. Math. Phys. 120, 1164 (1999) hep-th/0002062.

[15] S. J. Brodsky, J. R. Hiller and G. McCartor, Phys. Rev. D 60 (1999) 054506 hep-ph/9903388.

[16] A. Harindranath and R. Kundu, Phys. Rev. D 59 (1999) 116013.

[17] J. R. Hiller and S. J. Brodsky, Phys. Rev. D 59 (1999) 016006.

[18] L. Okun and I. Yu. Kobsarev, ZhETF, 43 (1962) 1904 (English translation: JETP 16 (1963) 1343); L. Okun, in proceedings of the 4th International Conference on Elementary Particles, Heidelberg, Germany (1967), edited by H. Filthuth, North-Holland (1968).

[19] I. Yu. Kobsarev and V. I. Zakharov, Ann. Phys. 60 (1970) 448.

[20] O. V. Teryaev, hep-ph/9904376 (1999).

[21] X.-S. Chen, D. Qing, and F. Wang, Chin. Phys. Lett. 16 (1999) 403, hepph/9802347; D. Qing, X.-S. Chen, and F. Wang, Phys. Rev. D 58 (1998) 114032.

[22] S. J. Brodsky and F. Schlumpf, Phys. Lett. B 329 (1994) 111.

[23] S. J. Brodsky, R. Roskies and R. Suaya, Phys. Rev. D8, 4574 (1973).

[24] F. E. Close, Phys. Lett. B 43 (1973) 422. 
[25] S. J. Brodsky and J. R. Hiller, Phys. Rev. D 46 (1992) 2141.

[26] S. J. Brodsky, J. R. Hiller and G. McCartor, Phys. Rev. D 58 (1998) 025005.

[27] P. V. Landshoff, J C. Polkinghorne and R. D. Short, Nucl. Phys. B 28, 225 (1971).

[28] S. J. Brodsky, F. E. Close, and J. F. Gunion, Phys. Rev. D 8 (1973) 3678.

[29] B.-Q. Ma, J. Phys. G 17 (1991) L53; B.-Q. Ma and Q.-R. Zhang, Z. Phys. C 58 (1993) 479.

[30] B.-Q. Ma and I. Schmidt, Phys. Rev. D 58 (1998) 096008.

[31] P. Hägler and A. Schäfer, Phys. Lett. B 430 (1998) 179.

[32] B.-Q. Ma, Phys. Lett. B 375 (1996) 320.

[33] B.-Q. Ma, I. Schmidt, and J. Soffer, Phys. Lett. B 441 (1998) 461. 